\title{
Insect galls of São Tomé das Letras (MG, Brazil)
}

\author{
Valéria Cid Maia ${ }^{1,2}$ \\ ${ }^{1}$ Museu Nacional, Quinta da Boa Vista, São Cristóvão, CEP 20940-040, Rio de Janeiro, RJ, Brasil \\ ${ }^{2}$ Corresponding author: Valéria Cid Maia, e-mail: maiavcid@acd.ufrj.br
}

MAIA, V.C. Insect galls of São Tomé das Letras (MG, Brazil). Biota Neotrop. 13(4): http://www.biotaneotropica. org.br/v13n4/en/abstract?article+bn03213042013

Abstract: Six localities of São Tomé das Letras (MG, Brazil) were investigated from September, 2011 to June, 2012. The local vegetation was examined in search of insect galls. A total of 152 morphotypes of insect galls were found on 94 plant species (74 genera and 37 families). Fabaceae, Melastomataceae, Myrtaceae, and Asteraceae were the plant families with the greatest richness of galls, with 20,18, 17, and 12 gall morphotypes, respectively. The super host genera were Copaifera L. (Fabaceae), Myrcia DC. ex. Guill. (Myrtaceae), and Miconia Ruiz \& Pav. (Melastomataceae), with 10, 10 and 09 gall morphotypes, respectively. The super host species was Copaifera cf.langsdorfii Desf. (Fabaceae), with 10 gall morphotypes. Galls were found on leaves, stems, buds, and aerial roots. Leaves were the most galled plant organ, followed by stems, and buds. The inducers belong to Diptera, Lepidoptera, Hemiptera, Thysanoptera, Coleoptera, and Hymenoptera, being Cecidomyiidae (Diptera) the most frequent and diversified gallers. The associated fauna included parasitoids (Hymenoptera), inquilines (Lepidoptera and Thysanoptera), successors (Formicidae, Hymenoptera), and predators (pseudoscorpion), obtained from 18, 02, 02, and 01 gall morphotype, respectively. Ten galling species are recorded for the first time in São Tomé das Letras (MG). The present study indicates São Tomé das Letras (MG) as an area of great richness of insect galls. Keywords: Atlantic forest, gall richness, host plants, insect-plant interaction.

MAIA, V.C. Galhas de insetos de São Tomé das Letras (MG, Brazil). Biota Neotrop. 13(4): http://www. biotaneotropica.org.br/v13n4/pt/abstract?article+bn03213042013

Resumo: Seis localidades de São Tomé das Letras (MG, Brasil) foram investigadas de setembro, 2011 a junho, 2012. A vegetação local foi examinada à procura de galhas entomógenas. Um total de 152 morfotipos de galhas de insetos foi encontrado em 94 espécies vegetais (74 gêneros e 37 famílias). Fabaceae, Melastomataceae, Myrtaceae e Asteraceae foram as famílias botânicas com maior riqueza de galhas, com 20, 18, 17 e 12 morfotipos de galha cada, respectivamente. Os gêneros super-hospedeiros foram Copaifera L. (Fabaceae), Myrcia DC. ex. Guill. (Myrtaceae) e Miconia Ruiz \& Pav. (Melastomataceae), com 10, 10 e 09 morfotipos de galhas. A espécie superhospedeira foi Copaifera langsdorfii Desf. (Fabaceae) com 10 morfotipos de galhas. Galhas foram encontradas em folhas, caules, gemas e raízes aéreas. As folhas foram o órgão vegetal mais atacado, seguidas por caules e gemas. Os galhadores pertencem às ordens Diptera, Lepidoptera, Hemiptera, Thysanoptera, Coleoptera e Hymenoptera, sendo os insetos da família Cecidomyiidae (Diptera) os indutores mais frequentes e diversificados. A fauna associada incluiu parasitóides (Hymenoptera), inquilinos (Lepidoptera e Thysanoptera), sucessores (Formicidae, Hymenoptera) e predadores (pseudoscorpion), obtidos de 18, 02,02 e 01 morfotipo de galha, respectivamente. Dez espécies galhadoras são registradas pela primeira vez para São Tomé das Letras (MG). O presente estudo indica São Tomé das Letras (MG) como uma área de grande riqueza de galhas de insetos.

Palavras-chave: Mata Atlântica, riqueza de galhas, plantas hospedeiras, interação inseto-planta. 


\section{Introduction}

Insect galls are pathologically developed cells, tissues or organs of plants that have risen mostly by hypertrophy (over-growth) and hyperplasy (cell proliferation) under the influence of these parasitic organisms (Shorthouse \& Rohfritsch 1992, Shorthouse et al. 2005). In this biological association, the galling species apparently derives all the benefit and the plant suffers loss of substance, deviations in the direction of growth, disturbances in sap flow, premature decays, and increase of non-essential parts at the cost of essential and other injury (Felt 1940, Stone \& Schönrogge 2003).

Several insect gall inventories have been developed in State of Minas Gerais (Fernandes et al. 1988, 1997, Urso-Guimarães et al. 2003, Maia \& Fernandes 2004, Carneiro et al. 2009, Coelho et al. 2009, Malves \& Frieiro-Costa 2012, Maia 2012). These inventories focused mainly on cerrado physiognomies, being the Atlantic Forest areas of Minas Gerais still little investigated.

The Atlantic Forest comprises high species diversity and richness, as well as a high number of flora and fauna endemisms. In the past, it covered approximately 330 million acres, but the original area has been greatly reduced and fragmented, remaining about $7 \%$ only. It has been considered a world biosphere reserve and a high priority area for biological conservation (Myers et al. 2000).

The main objective of this study is to contribute to the knowledge of the richness of insect galls from Atlantic Forest areas of Minas Gerais.

\section{Material and Methods}

Insect galls were collected seasonally (from September, 2011 to June, 2012) in six localities of São Tomé das Letras (MG): Ladeira do Amendoim (22 42' 24' S and 44 58' 57' W), Cachoeira da Lua ( $21^{\circ} 42^{\prime} 11^{\prime \prime} \mathrm{S}$ and $\left.44^{\circ} 56^{\prime} 24^{\prime \prime} \mathrm{W}\right)$, Vale das Borboletas (22 $42^{\prime} 24^{\prime \prime}$

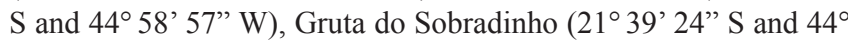

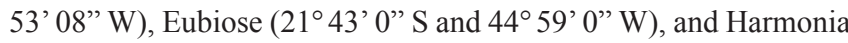
$\left(21^{\circ} 43^{\prime} 24^{\prime \prime} \mathrm{S}\right.$ and $\left.44^{\circ} 57^{\prime} 33^{\prime \prime} \mathrm{W}\right)$.

The vegetation was examined in search of galling insects during two hours in each locality per collection. All plant organs were investigated, except for subterranean roots. Galled plants, preferably with flowers and fruits were pressed for identification and preservation. All gall morphotypes were photographed in field and characterized by shape, color, presence of trichomes, and plant organ.

Samples of each morphotype were collected and transported individually in labeled plastic bags. Larvae and pupa of immature insects were obtained in the laboratory from the dissection of each gall morphotype under a stereoscopic microscope. This procedure also allowed the determination of the number of internal chambers. The pupal exuviae and adults were obtained from rearing, by keeping samples of each kind of gall individually in covered plastic pots with damp cotton at the bottom. These pots were examined daily for adults' emergence. The galls were kept in these pots until their deterioration.

All insects were preserved in $70 \%$ alcohol. The gall midges (larvae, pupae, pupal exuviae and adults) were later mounted on microscope slides following the methodology of Gagné (1989). The Cecidomyiidae genera were identified based on the keys of Gagné (1994). The insects were incorporated in the entomological collection of Museu Nacional, Universidade Federal do Rio de Janeiro (MNRJ).

The plant species were identified by Dr. Gracialda Costa Ferreira (Universidade Federal Rural da Amazônia, Brazil), and the dried specimens were incorporated into the herbarium of the Instituto de Ciências Agrárias (Pará, Brazil).

\section{Results}

A total of 152 gall morphotypes were found in São Tomé das Letras (MG). They occurred on 94 plant species distributed in 74 genera and 37 families (Table 1). The medium number of gall morphotypes per plant species was 1.62 .

Fabaceae, Melastomataceae, Myrtaceae, and Asteraceae were the plant families with the greatest richness of galls, with 20, 18, 17, and 12 morphotypes each, respectively. The super host genera were Copaifera L. (Fabaceae), Myrcia DC. ex. Guill. (Myrtaceae), and Miconia Ruiz \& Pav. (Melastomataceae), with 10, 10, and 9 gall morphotypes. The super host species were Copaifera langsdorfii Desf. (Fabaceae), Myrcia sylvatica (G. Mey) DC. (Myrtaceae), and Calophyllum brasiliense Cambéss (Calophyllaceae), with 10, 7, and 6 gall morphotypes each, respectively. Galls were found on leaves (79 morphotypes, ca. 57\%), stems (41 morphotypes, ca. 28\%), buds (21 morphotypes, ca. 14\%), and aerial roots (01 morphotype, ca. $0.7 \%$ ). Leaves were the most galled plant organ, followed by stems, and buds. Flower, fruit and tendril galls were not found. Five morphotypes occurred on leaves and buds (ca.3\%) and four morphotypes on buds and stems (ca. 2.6\%).

The inducers are represented by six insect orders: Diptera $(82$ gall morphotypes, ca. 86\%), Lepidoptera (05 gall morphotypes, ca. 5\%), Hemiptera (04 gall morphotypes, ca. 4\%), Thysanoptera (02 gall morphotypes, ca. 2\%), Coleoptera (01 gall morphotype, ca. 1\%), and Hymenoptera (01 gall morphotype, ca. 1\%), being Cecidomyiidae (Diptera) the most frequent and diversified gallers (81 gall morphotypes, ca. $85 \%$ ).

Concerning gall morphology, the following shapes were found: bulbous, burn-shaped, bursiform, catterpillar-like, circular, conical, cylindrical, discoid, elliptical, fusiform, globoid, horn-shaped, imbricated, leaf roll, linear, marginal leaf roll, ovoid, rosette, rugose, spot-like, and vermiform. Globoid and fusiform galls predominate with ca. $34 \%$ and ca. $27 \%$, respectively, followed by circular ones (9\%), marginal leaf roll (ca. 4\%), bulbous, ovoid, conical and leaf roll (ca.3\% each), and cylindrical (ca 2.5\%). The other shapes were represented by less than $2 \%$ each. The majority of the galls were glabrous (86.18\%) and one-chambered (90.8\%).

The associated fauna included parasitoids (Hymenoptera), inquilines (Lepidoptera and Thysanoptera), successors (Formicidae, Hymenoptera), and predators (pseudoscorpion), obtained from 18, 02,02 , and 01 gall morphotype, respectively. Ten galling species are recorded for the first time in São Tomé das Letras (MG): Asphondylia serrata Maia, 2004; Contarinia gemmae Maia, 2003; Dactylodiplosis heptaphylli Maia, 2004; Lopesia caulinaris Maia, 2003; Lopesia conspicua Maia, 2003; Lopesia elliptica Maia, 2003; Lopesia linearis Maia, 2003; Lopesia similis Maia, 2004; Myrciaryiamia admirabilis Maia, 2007; and Tomoplagia rudolphi (Lutz \& Lima, 1918). All gall records are new as this is the first gall inventory of São Tomé das Letras (MG).

Data on insect galls are presented under host plant family, genus and species in alphabetical order. They include morphological characterization (plant organ, shape, color, presence/absence of trichomes, number of internal chamber), galler, locality and date of collection, associated fauna, and previous records in Brazil. The values in parenthesis indicate the number of gall morphotypes.

Anacardiaceae $(\mathrm{n}=1)$

Tapirira $\mathrm{sp} .(\mathrm{n}=1)$

Leaf gall, circular, yellow, glabrous, one-chambered (Figure 1). Galler: Hemiptera. Locality: Cachoeira da Lua. Date: September/2011. Previous records: Maia et al. (2008) described other gall on Tapirira guianensis Aubl. from Bertioga (SP). 
Maia, V.C.

Table 1. Distribution of insect gall morphotypes by plant families and species in São Tomé das Letras (MG, Brazil).

\begin{tabular}{|c|c|c|}
\hline Plant family $(n=37)$ & Plant species $(n=94)$ & Number of insect gall morphotypes $(n=152)$ \\
\hline \multirow[t]{2}{*}{ Anacardiaceae } & Tapirira $\mathrm{sp}$ & 01 \\
\hline & & Total 01 \\
\hline \multirow[t]{3}{*}{ Annonaceae } & Duguetia furfuracea & 01 \\
\hline & Pseudoxandra sp. & 01 \\
\hline & & Total 02 \\
\hline \multirow[t]{7}{*}{ Asteraceae } & Baccharis sp.1 & 03 \\
\hline & Baccharis cfr & 01 \\
\hline & Dimerostemma brasilianum & 01 \\
\hline & Eremanthus polycephalus & 04 \\
\hline & Vernonia sp. & 02 \\
\hline & Not determined & 01 \\
\hline & & Total 12 \\
\hline \multirow[t]{4}{*}{ Bignoniaceae } & Macfadyena sp. & 01 \\
\hline & Stigophyllum riparium & 01 \\
\hline & Not determined & 01 \\
\hline & & Total 03 \\
\hline \multirow[t]{2}{*}{ Burseraceae } & Protium heptaphyllum & 04 \\
\hline & & Total 04 \\
\hline \multirow[t]{2}{*}{ Calophyllaceae } & Calophyllum brasiliense & 06 \\
\hline & & Total 06 \\
\hline \multirow[t]{3}{*}{ Celastraceae } & Maytenus sp. & 01 \\
\hline & Hippocratea sp. & 01 \\
\hline & & Total 02 \\
\hline \multirow[t]{2}{*}{ Clusiaceae } & Kielmeyera sp. & 01 \\
\hline & & Total 01 \\
\hline \multirow[t]{2}{*}{ Dilleniaceae } & Davilla brasiliensis & 02 \\
\hline & & Total 02 \\
\hline \multirow[t]{2}{*}{ Ebenaceae } & Diospyros sp. & 01 \\
\hline & & Total 01 \\
\hline \multirow[t]{2}{*}{ Erythroxylaceae } & Erythroxylum cf. suberosum & 05 \\
\hline & & Total 05 \\
\hline \multirow[t]{3}{*}{ Euphorbiaceae } & Croton sp. & 03 \\
\hline & Pera sp. & 01 \\
\hline & & Total 04 \\
\hline \multirow[t]{8}{*}{ Fabaceae } & Andira $\mathrm{sp}$ & 03 \\
\hline & Copaifera cf. langsdorfii & 10 \\
\hline & Dalbergia foliosa & 03 \\
\hline & Inga crassifólia & 01 \\
\hline & Inga edulis & 01 \\
\hline & Machaerium sp. & 01 \\
\hline & Stylosanthes gracilis & 01 \\
\hline & & Total 20 \\
\hline \multirow[t]{3}{*}{ Lamiaceae } & Hyptis recurvata & 01 \\
\hline & Ocimum sp. & 01 \\
\hline & & Total 02 \\
\hline \multirow[t]{3}{*}{ Lauraceae } & Ocotea cernua & 01 \\
\hline & Ocotea sp. & 01 \\
\hline & & Total 02 \\
\hline \multirow[t]{2}{*}{ Loranthaceae } & Struthanthus sp. & 01 \\
\hline & & Total 01 \\
\hline \multirow[t]{2}{*}{ Lythraceae } & Cuphea sp. & 01 \\
\hline & & Total 01 \\
\hline Malpighiaceae & $\begin{array}{c}\text { Byrsonima verbascifolia (L.) Rich. var. } \\
\text { intermedia }\end{array}$ & 01 \\
\hline
\end{tabular}


Table 1. Continued...

\begin{tabular}{|c|c|c|}
\hline Plant family $(n=37)$ & Plant species $(n=94)$ & Number of insect gall morphotypes $(n=152)$ \\
\hline \multirow{9}{*}{ Malvaceae } & $\begin{array}{c}\text { Byrsonima verbascifolia (L.) Rich. var. } \\
\text { villosa }\end{array}$ & 01 \\
\hline & Byrsonima verbascifolia & 01 \\
\hline & Mascagnia sp. & 01 \\
\hline & Mendocia sp. & 01 \\
\hline & & Total 05 \\
\hline & Luehea grandiflora & 01 \\
\hline & Melochia sp. & 01 \\
\hline & Waltheria indica & 01 \\
\hline & & Total 03 \\
\hline \multirow[t]{14}{*}{ Melastomataceae } & Aciotis cf. indecora & 02 \\
\hline & Clidemia sp.1 & 02 \\
\hline & Clidemia sp.2 & 01 \\
\hline & Miconia ligustroides & 01 \\
\hline & Miconia cf. theaezans & 01 \\
\hline & Miconia sp.1 & 03 \\
\hline & Miconia sp.2 & 01 \\
\hline & Miconia sp.3 & 02 \\
\hline & Mouriri sp. & 01 \\
\hline & Tibouchina granulosa & 01 \\
\hline & Tibouchina cf. stenocarpa & 01 \\
\hline & not determined 1 & 01 \\
\hline & not determined 2 & 01 \\
\hline & & Total 18 \\
\hline \multirow[t]{2}{*}{ Monimiaceae } & Moriri sp. & 01 \\
\hline & & Total 01 \\
\hline \multirow[t]{3}{*}{ Moraceae } & Brosimum sp. & 02 \\
\hline & Pseudolmedia macrophylla & 03 \\
\hline & & Total 05 \\
\hline \multirow[t]{3}{*}{ Myrsinaceae } & Rapanea cf. parvifolia & 01 \\
\hline & Rapanea sp. & 01 \\
\hline & & Total 02 \\
\hline \multirow[t]{10}{*}{ Myrtaceae } & Aulomyrcia tomentosa var. longipes & 01 \\
\hline & Campomanesia pubescens & 01 \\
\hline & Eugenia biflora & 01 \\
\hline & Eugenia punicifolia & 01 \\
\hline & Eugenia $\mathrm{sp}$ & 01 \\
\hline & Myrcia splendens & 02 \\
\hline & Myrcia sylvatica & 07 \\
\hline & Myrcia sp. & 01 \\
\hline & Myrciaria floribunda & 02 \\
\hline & & Total 17 \\
\hline \multirow[t]{3}{*}{ Nyctaginaceae } & Guapira sp. & 01 \\
\hline & Neea sp. & 02 \\
\hline & & Total 03 \\
\hline \multirow[t]{2}{*}{ Passifloraceae } & Turnera sp. & 01 \\
\hline & & Total 01 \\
\hline \multirow[t]{2}{*}{ Piperaceae } & Piper sp. & 01 \\
\hline & & Total 01 \\
\hline \multirow[t]{2}{*}{ Proteaceae } & Roupala montana Aubl. var. montana & 02 \\
\hline & & Total 02 \\
\hline Rubiaceae & Amaioua intermedia var. brasiliana & 02 \\
\hline
\end{tabular}


Maia, V.C.

Table 1. Continued...

\begin{tabular}{|c|c|c|}
\hline Plant family $(n=37)$ & Plant species $(n=94)$ & Number of insect gall morphotypes $(n=152)$ \\
\hline \multirow{9}{*}{ Sapindaceae } & Coussarea $\mathrm{sp}$ & 01 \\
\hline & Faramea sp. & 01 \\
\hline & Palicourea rigida & 01 \\
\hline & Sabicea brasiliensis & 01 \\
\hline & & Total 06 \\
\hline & Serjania communis & 02 \\
\hline & Serjania glutinosa & 01 \\
\hline & Serjania meridionalis & 01 \\
\hline & Serjania sp. & 01 \\
\hline \multirow{3}{*}{ Sapotaceae } & & Total 05 \\
\hline & Manilkara sp. & 01 \\
\hline & & Total 01 \\
\hline \multirow[t]{2}{*}{ Siparunaceae } & Siparuna guianensis & 01 \\
\hline & & Total 01 \\
\hline \multirow[t]{2}{*}{ Smilacaceae } & Smilax cissoides & 01 \\
\hline & & Total 01 \\
\hline \multirow[t]{3}{*}{ Solanaceae } & Solanum lycocarpum & 01 \\
\hline & Solanum sp.1 & 01 \\
\hline & Solanum sp.2 & 01 \\
\hline \multirow{4}{*}{ Sterculiaceae } & & Total 03 \\
\hline & Buettneria sp & 01 \\
\hline & Not determined & 01 \\
\hline & & Total 02 \\
\hline \multirow[t]{3}{*}{ Verbenaceae } & Lantana camara & 01 \\
\hline & Lantana sp. & 01 \\
\hline & & Total 02 \\
\hline \multirow[t]{3}{*}{ Vochysiaceae } & cf. Salvertia convallariodora & 01 \\
\hline & Vochysia cf. maxima & 03 \\
\hline & & Total 04 \\
\hline
\end{tabular}

Annonaceae $(\mathrm{n}=2)$

Duguetia furfuracea (A. St.-Hil) Saff. (n=1)

Leaf gall, globoid, yellow, glabrous, one-chambered (Figure 2). Galler: not determined. Other dwellers: parasitoids (Hymenoptera). Localities: Ladeira do Amendoim, Cachoeira da Lua. Date: December/2011, March/2012.

Previous records: Vale do Jequitinhonha, MG (Fernandes et al. 1997), Delfinópolis, MG (Urso-Guimarães et al. 2003), Santa Rita do Passa Quatro, SP (Urso-Guimarães \& Scareli-Santos 2006), and Ingaí, MG (Malves \& Frieiro-Costa 2012). Saito \& Urso-Guimarães (2012) described other galls on this host plant from Luiz Antonio (SP).

\section{Pseudoxandra sp. $(\mathrm{n}=1)$}

Bud gall, conical, green, glabrous, one-chambered (Figure 3). Galler: not determined. Locality: Eubiose. Date: June/2012.

Asteraceae $(\mathrm{n}=12)$

Baccharis sp. $1(\mathrm{n}=3)$

Leaf gall, circular, yellow, glabrous, one-chambered (Figure 4). Galler: Cecidomyiidae (Diptera). Locality: Cachoeira da Lua. Date: September/2011.

Leaf gall, cylindrical, green, glabrous, one-chambered. Galler: Cecidomyiidae (Diptera). Locality: Cachoeira da Lua. Date: September/2011.
Stem swelling, fusiform, brown, glabrous, one-chambered. Galler: Cecidomyiidae (Diptera). Locality: Cachoeira da Lua. Date: September/2011.

Baccharis cfr. $(\mathrm{n}=1)$

Leaf or bud gall, conical or ovoid, green, glabrous, onechambered (Figure 5). Galler: Cecidomyiidae (Diptera). Locality: Vale das Borboletas. Date: June/2012.

Previous records: Maia \& Fernandes (2004), Maia et al. (2008), Coelho et al. (2009), Carneiro et al. (2009), Maia \& Souza (in press), Maia et al. (in press), and Maia (2012) described several galls on Baccharis spp. from Serra de São José (MG), Bertioga (SP), Serra do Cipó (MG), Cadeia do Espinhaço (MG), Ilha do Cabo Frio (Arraial do Cabo, RJ), Santa Teresa (ES), and Itamonte (MG), respectively.

Dimerostemma brasilianum Cass. $(\mathrm{n}=1)$

Bud gall, fusiform, brown, glabrous, multi-chambered (Figure 6). Galler: not determined. Locality: Ladeira do Amendoim. Date: December/2011.

Eremanthus polycephalus (DC.) MacLeish. (n=4)

Leaf gall, globoid, yellow, micropubescent, one-chambered (Figure 7). Galler: Asphondylia serrata Maia, 2004 (Diptera, Cecidomyiidae). Localities: Ladeira do Amendoim, Vale das Borboletas, Harmonia, Eubiose, Gruta do Sobradinho. Date: September/2011, December/2011, March/2012, June/2012. Previous 

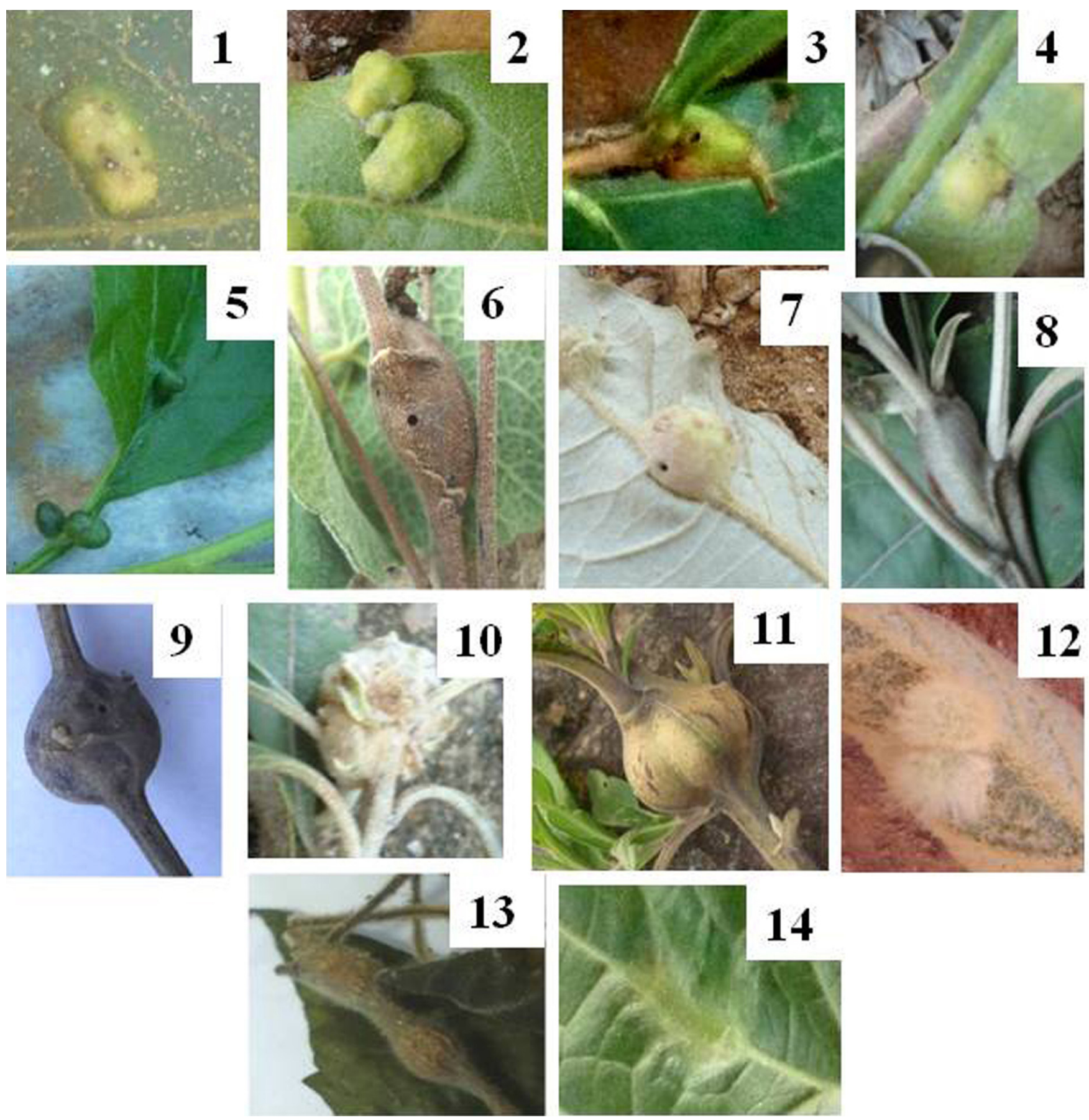

Figures 1-14. Insect galls from São Tomé das Letras: 1. Leaf gall on Tapirira sp.; 2. Leaf gall on Duguetia furfuracea; 3. Bud gall on Pseudoxandra sp.; 4. Leaf gall on Baccharis sp.; 5. Leaf or bud gall on Baccharis cfr.; 6. Bud gall on Dimerostemma brasilianum; 7-10. Galls on Eremanthus polycephalus: 7. Leaf gall, 8. Stem swelling, 9. Stem swelling, 10. Bud gall; 11. Stem gall on Vernonia sp.; 12. Leaf gall on Asteraceae not determined; 13. Stem swelling on Macfadyena sp.; 14. Stem swelling on Stigophyllum riparium.

records: Vale do Jequitinhonha (Fernandes et al. 1997), Serra de São José (Maia \& Fernandes 2004), and Ingaí, MG (Malves \& FrieiroCosta 2012).

Stem swelling, fusiform, glabrous, one-chambered (Figure 8). Galler: not determined. Locality: Gruta do Sobradinho. Date: March/2012, June/2012. Previous records: Vale do Jequitinhonha (Fernandes et al. 1997), and Cadeia do Espinhaço, MG (Carneiro et al. 2009).
Stem swelling, globoid, brown, glabrous, one-chambered (Figure 9). Galler: Cecidomyiidae (Diptera). Other dwellers: successors - ants (Formicidae, Hymenoptera). Localities: Ladeira do Amendoim, Cachoeira da Lua, Harmonia, Eubiose, Gruta do Sobradinho. Date: September/2011, December/2011, March/2012, June/2012. Previous records: Vale do Jequitinhonha, MG (Fernandes et al. 1997), Cadeia do Espinhaço, MG (Carneiro et al. 2009), and Ingaí, MG (Malves \& Frieiro-Costa 2012). 
Bud gall, globoid, glabrous, one-chambered (Figure 10). Galler: not determined. Locality: Gruta do Sobradinho. Date: March/2012.

Vernonia sp. $(\mathrm{n}=2)$

Stem gall, bulbous, brown, glabrous, one-chambered (Figure 11). Galler: Tomoplagia rudolpphi (Tephritidae, Diptera). Localities: Cachoeira da Lua, Ladeira do Amendoim. Date: September/2011, March/2012, June/2012.

Previous records: Fernandes et al. (1988), Urso-Guimarães et al. (2003), Maia \& Fernandes (2004), and Maia (2012) recorded the same gall from Belo Horizonte, Delfinópolis, Serra de São José, and Itamonte (MG), respectively.

Leaf gall, globoid, green, glabrous, one-chambered. Galler: not determined. Locality: Cachoeira da Lua. Date: March/2012, June/2012.

Other galls on Vernonia spp. have been recorded by Fernandes et al. (1997) from Vale do Jequitinhonha (MG), Maia (2001) from Carapebus (RJ), Fernandes \& Negreiros (2006) from Aimorés (MG), Maia et al. (2008) from Bertioga (SP), and Maia et al. (in press) from Santa Teresa (ES).

Asteraceae não determinada $(n=1)$

Leaf gall, globoid, yellowish, micropubescent, one-chambered (Figure 12). Galler: Cecidomyiidae (Diptera). Locality: Ladeira do Amendoim. Date: September/2011, March/2012.

Bignoniaceae $(\mathrm{n}=3)$

Macfadyena $\mathrm{sp} .(\mathrm{n}=1)$

Stem swelling, globoid, brown, glabrous, one-chambered (Figure 13). Galler: Cecidomyiidae (Diptera). Locality: Vale das Borboletas. Date: March/2012.

Stigophyllum riparium (Kunth) Sandwith ( $\mathrm{n}=1)$

Stem swelling, fusiform, brown, glabrous, one-chambered (Figure 14). Galler: not determined. Locality: Eubiose. Date: June/2012

Bignoniaceae não determinada $(\mathrm{n}=1)$

Vein swelling, fusiform, brown, woody, one-chambered (Figure 15). Galler: Cecidomyiidae (Diptera). Other dwellers: parasitoids (Hymenoptera). Locality: Cachoeira da Lua. Date: September/2011.

Burseraceae $(n=4)$

\section{Protium heptaphyllum (Aubl.) March ( $\mathrm{n}=4)$}

Leaf gall, circular, red, glabrous, one-chambered (Figure 16). Galler: Hemiptera. Localities: Vale das Borboletas, Cachoeira da Lua, Gruta do Sobradinho. Date: September/2011, December/2011, March/2012, June/2012. Previous records: Vale do Rio Doce, MG (Fernandes et al. 2001).

Marginal leaf roll, green, glabrous, one-chambered (Figure 17). Galler: Lopesia similis Maia, 2004 (Diptera, Cecidomyiidae). Localities: Vale das Borboletas, Gruta do Sobradinho. Date: September/2011, December/2011, June/2012. Previous records: Carapebus, RJ (Maia 2001), Ilha do Cabo Frio (Arraial do Cabo, RJ (Maia \& Souza, in press), and Itamonte, MG (Maia 2012)).

Leaf gall, cylindrical, green or yellowish, glabrous, onechambered (Figure 18). Galler: Dactylodiplosis heptaphylli Maia, 2004 (Diptera, Cecidomyiidae). Locality: Vale das Borboletas, Cachoeira da Lua, Gruta do Sobradinho. Date: September/2011, March/2012, June/2012.

Stem swelling, globoid, brown, glabrous, multi-chambered (Figure 19). Galler: not determined. Dweller: Hymenoptera.
Localities: Gruta do Sobradinho, Cachoeira da Lua. Date: September/2011, March/2012.

Previous records: Maia (2001), Santos et al. (2010), and Malves \& Frieiro-Costa (2012) recorded the other galls on Protium heptaphyllum from Carapebus (RJ), Goiânia (GO) and Ingaí (MG).

Calophyllaceae $(n=6)$

Calophyllum brasiliense Cambéss. ( $\mathrm{n}=6)$

Leaf gall, linear, green, glabrous, one-chambered (Figure 20). Galler: Lopesia linearis Maia, 2003 (Cecidomyiidae, Diptera). Localities: Gruta do Sobradinho, Vale das Borboletas. Date: September/2011, December/2011, March/2012. Previous records: Serra de São José (Maia \& Fernandes 2004).

Leaf gall, elliptical, green, glabrous, one-chambered (Figure 21). Galler: Lopesia elliptica Maia, 2003 (Cecidomyiidae, Diptera).Galler: Lopesia elliptica Maia, 2003 (Cecidomyiidae, Diptera). Locality: Vale das Borboletas. Date: September/2011, December/2011. Previous records: Serra de São José, MG (Maia \& Fernandes 2004), and Bertioga, SP (Maia et al. 2008).

Stem swelling, fusiform, brown, glabrous, one-chambered (Figure 22). Galler: Lopesia caulinaris Maia, 2003 (Cecidomyiidae, Diptera). Localities: Gruta do Sobradinho, Vale das Borboletas. Date: September/2011, December/2011, March/2012, June/2012. Previous records: Serra de São José (Maia \& Fernandes 2004), and Bertioga, SP (Maia et al. 2008).

Bud gall, fusiform, brown, glabrous, one or multi-chambered (Figure 23). Galler: Contarinia gemmae Maia, 2003 (Cecidomyiidae, Diptera). Other dwellers: predators (Pseudoscorpion) and parasitoids (Hymenoptera). Localities: Gruta do Sobradinho, Vale das Borboletas. Date: September/2011, December/2011, March/2012. Previous records: Bertioga, SP (Maia et al. 2008).

Leaf gall, globoid, glabrous, one-chambered (Figure 24). Galler: Lopesia conspicua Maia, 2003 (Cecidomyiidae, Diptera). Localities: Vale das Borboletas, Gruta do Sobradinho. Date: September/2011, December/2011, June/2012.

Marginal leaf roll, green, glabrous, one-chambered (Figure 25). Galler: Cecidomyiidae (Diptera). Localities: Gruta do Sobradinho, Vale das Borboletas. Date: December/2011, June/2012. Previous records: Serra de São José (Maia \& Fernandes 2004).

Celastraceae $(n=2)$

Maytenus sp. $(\mathrm{n}=1)$

Leaf gall, globoid, red, glabrous, one-chambered (Figure 26). Galler: Cecidomyiidae (Diptera). Locality: Vale das Borboletas. Date: September/2001, December/2011, March/2012, June/2012.

Previous records: Galls on Maytenus spp. have been recorded by Maia (2001) from Carapebus and Maricá (RJ), Oliveira \& Maia (2005) from Grumari (Rio de Janeiro, RJ), and Maia et al. (2008) from Bertioga (SP)

Hippocratea sp. $(\mathrm{n}=1)$

Leaf gall, circular, brown, glabrous, one-chambered (Figure 27). Galler: not determined. Locality: Vale das Borboletas. Date: September/2011.

Clusiaceae $(\mathrm{n}=1)$

Kielmeyera $\mathrm{sp} .(\mathrm{n}=1)$

Leaf gall, circular, yellowish, glabrous, one-chambered (Figure 28). Galler: Cecidomyiidae (Diptera). Locality: Ladeira do Amendoim. Date: December/2011, March/2012. 

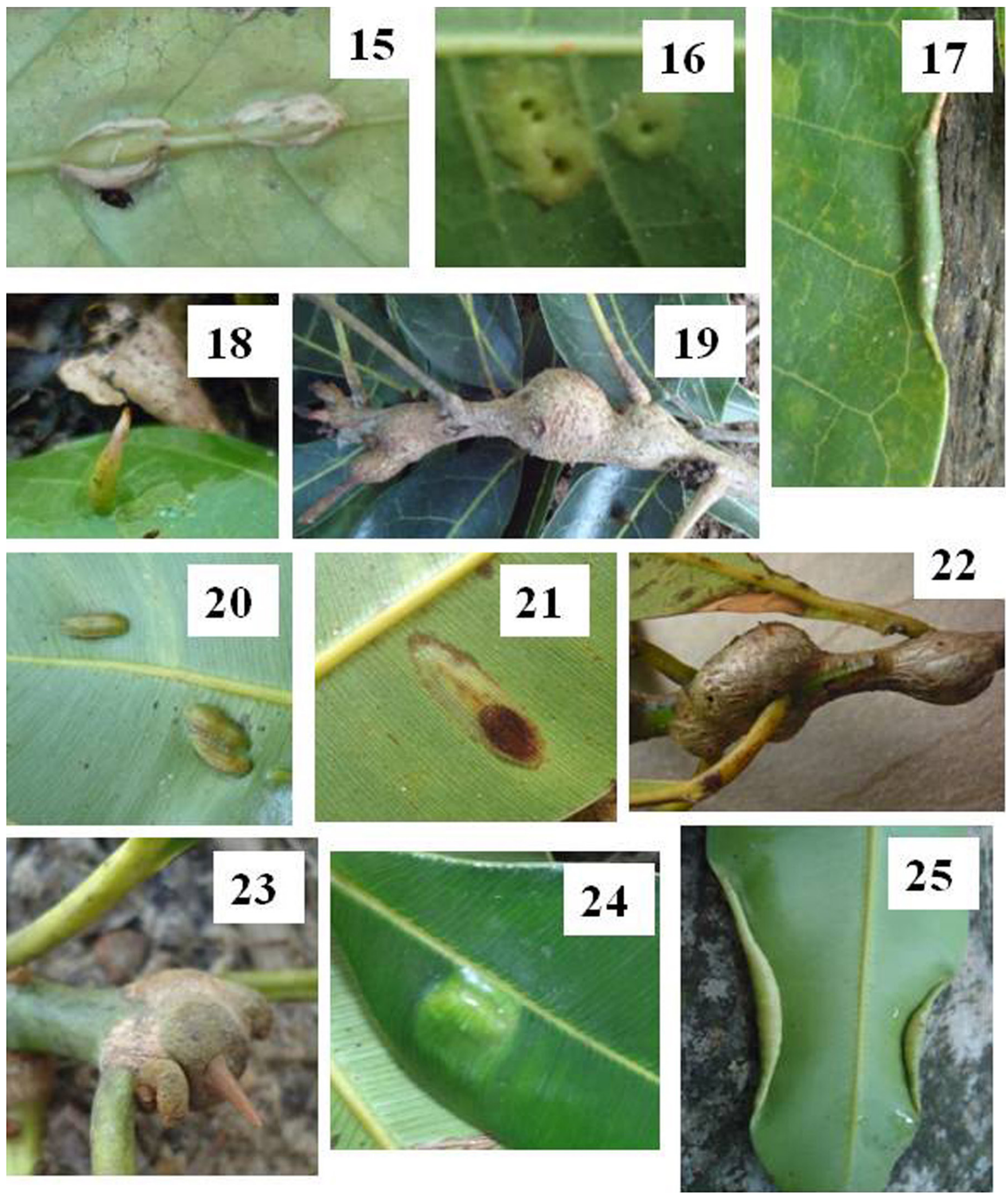

Figures 15-25. Insect galls from São Tomé das Letras: 15. Vein swelling on Bignoniaceae not determined; 16-19. Galls on Protium heptaphyllum: 16. Circular leaf gall, 17. Marginal roll, 18. Cylindrical leaf gall, 19. Stem swelling; 20-25 Galls on Calophyllum brasiliense: 20. Linear leaf gall, 21. Elliptical leaf gall, 22. Stem gall, 23. Bud gall; 24. Globoid gall, 25. Marginal roll.. 
Dilleniaceae $(\mathrm{n}=2)$

\section{Davilla brasiliana DC. $(\mathrm{n}=2)$}

Leaf gall, circular, yellowish, glabrous, one-chambered (Figure 29). Galler: Cecidomyiidae (Diptera). Locality: Ladeira do Amendoim. Date: March/2012.

Bud gall, imbricated, green or brown, glabrous (Figure 30). Galler: Asphondylia sp. (Diptera, Cecidomyiidae). Date: September/2011, December/2011, June/2012. Localities: Cachoeira da Lua, Gruta do Sobradinho, Eubiose. Previous records: Serra de São José, MG (Maia \& Fernandes 2004).

Other galls on Davilla spp. have been described by Fernandes et al. (1997), Fernandes et al. (2001), and Maia et al. (in press) from Vale do Jequitinhonha (MG), Vale do Rio Doce (MG), and Santa Teresa (ES).

Ebenaceae $(\mathrm{n}=1)$

Diospyros $\mathrm{sp} .(\mathrm{n}=1)$

Stem swelling, globoid, brown, glabrous, one-chambered (Figure 31). Galler: not determined. Locality: Eubiose. Date: June/2012.

Erythroxylaceae $(n=5)$

Erythroxylum cf. suberosum A. St.-Hil. (n=5)

Leaf gall, red, hairy (Figure 32). Galler: Myrciaryiamia admirabilis Maia, 2007 (Cecidomyiidae, Diptera). Localities: Cachoeira da Lua, Gruta do Sobradinho, Vale das Borboletas. Date: December/2011, March/2012, June/2012.

Previous records: Serra de São José, MG (Maia \& Fernandes 2004), Santa Rita do Passa Quatro, SP (Urso-Guimarães \& ScareliSantos 2006) and Ingaí, MG (Malves \& Frieiro-Costa 2012).

Apical bud, globoid, brown, glabrous, multi-chambered (Figure 33). Galler: Hymenoptera. Locality: Ladeira do Amendoim. Date: December/2011, March/2012.

Marginal leaf roll, green, glabrous, one-chambered (Figure 34). Galler: Cecidomyiidae (Diptera). Localities: Gruta do Sobradinho, Ladeira do Amendoim. Date: June/2012.

Leaf gall globoid, green, glabrous, one-chambered (Figure 35). Galler: not determined. Locality: Ladeira do Amendoim. Date: December/2011, March/2012, June/2012.

Leaf roll, green, glabrous, one-chambered. Galler: Cecidomyiidae (Diptera). Other dwellers: inquiline (Lepidoptera). Localities: Cachoeira da Lua, Gruta do Sobradinho. Date: December/2011.

Other galls on Erythroxylum spp. have been described by Fernandes et al. (1988) from Belo Horizonte (MG), Fernandes et al. (1997) from Vale do Jequitinhonha (MG), Fernandes et al. (2001) from Vale do Rio Doce (MG), Maia (2001) from Carapebus and Maricá (RJ), Julião et al. (2002) from Pantanal sul mato-grossense (MS), Urso-Guimarães et al. (2003) from Delfinópolis (MG), Maia \& Fernandes (2004) from Serra de São José (MG), Oliveira \& Maia (2005) from Grumari (Rio de Janeiro, RJ), Maia et al. (2008) from Bertioga (SP), Maia \& Oliveira (2010) from Ilha Grande (Angra dos Reis, RJ), Maia \& Souza (in press) from Ilha do Cabo Frio (Arraial do Cabo, RJ), and Maia et al. (in press) from Santa Teresa (ES).

Euphorbiaceae $(n=4)$

Croton sp. $(\mathrm{n}=3)$

Leaf gall, globoid, yellowish, hairy, one-chambered (Figure 36). Galler: Cecidomyiidae (Diptera). Localities: Cachoeira da Lua, Vale das Borboletas, Eubiose. Date: September/2011, December/2011, June/2012. Previous records: Malves \& Frieiro-Costa (2012) recorded the same gall from Ingaí, MG.
Leaf gall, ovoid, yellowish or brownish, with few short trichomes (Figure 37), glabrous, one-chambered. Galler: Cecidomyiidae (Diptera). Other dwellers: parasitoids (Hymenoptera). Locality: Cachoeira da Lua. Date: December/2011. Previous records: Malves \& Frieiro-Costa (2012) recorded the same gall from Ingaí, MG.

Leaf gall, discoid, green, glabrous, one-chambered. Galler: Cecidomyiidae (Diptera). Locality: Eubiose. Date: June/2012.

Several galls on Croton sp. have been recorded by Fernandes et al. (2001), Urso-Guimarães et al. (2003), Maia \& Fernandes (2004), Carneiro et al. (2009), Malves \& Frieiro-Costa (2012), Maia \& Souza (in press), Maia et al. (in press), and Maia (2012) from Vale do Rio Doce (MG), Delfinópolis (MG), Serra de São José (MG), Cadeia do Espinhaço (MG), Ingaí (MG), Ilha do Cabo Frio (Arraial do Cabo, RJ), Santa Teresa (ES), and Itamonte (MG).

Pera sp. $(\mathrm{n}=1)$

Leaf gall, circular, green, glabrous, one-chambered (Figure 38). Galler: not determined. Locality: Vale das Borboletas. Cachoeira da Lua. Date: September/2011, December/2011.

Previous records: Galls on Pera spp. have been described by Fernandes et al. (2001) from Vale do Rio Doce (MG), and Maia et al. (2008) from Bertioga (SP).

Fabaceae $(n=20)$

Andira sp. $(\mathrm{n}=3)$

Leaf gall, vermiform, green, one-chambered (Figure 39). Galler: Cecidomyiidae (Diptera). Other dwellers: parasitoids (Hymenoptera). Localities: Vale das Borboletas, Gruta do Sobradinho. Date: September/2011, March/2012. Previous records: Serra de São José, MG (Maia \& Fernandes 2004), and Luiz Antonio, SP (Saito \& UrsoGuimarães 2012).

Leaf gall, globoid, yellow or reddish, glabrous, one-chambered (Figure 40). Galler: not determined. Locality: Cachoeira da Lua. Date: March/2012.

Marginal leaf roll, green, glabrous, one-chambered (Figure 41). Galler: not determined. Locality: Cachoeira da Lua. Date: March/2012.

Previous records: Fernandes et al. (1988), Fernandes et al. (2001), Julião et al. (2002), Maia et al. (2008), and Maia et al. (in press) recorded galls on Andira spp. from Belo Horizonte (MG), Vale do Rio Doce (MG), Pantanal sul mato-grossense (MS), Bertioga (SP), and Santa Teresa (ES), respectively.

\section{Copaifera cf. langsdorfii Desf. $(\mathrm{n}=10)$}

Midvein swelling, fusiform, brown, woody, glabrous, onechambered (Figure 42). Galler: Cecidomyiidae (Diptera). Localities: Vale das Borboletas, Cachoeira da Lua, Gruta do Sobradinho. Date: March/2011, September/2011, June/2012. Previous records: Belo Horizonte (Fernandes et al. 1988), and Vale do Jequitinhonha, MG (Fernandes et al. 1997).

Bud or leaf gall, horn-shaped, green or reddish, glabrous, onechambered (Figure 43). Galler: Cecidomyiidae (Diptera). Localities: Cachoeira da Lua, Gruta do Sobradinho. Date: March/2012, June/2012. Previous records: Belo Horizonte (Fernandes et al. 1988) and Serra de São José, MG (Maia \& Fernandes 2004).

Leaf gall, globoid, green, reddish or yellowish, glabrous, one-chambered (Figure 44). Galler: not determined. Localities: Ladeira do Amendoim, Vale das Borboletas, Gruta do Sobradinho, Cachoeira da Lua. Date: June/2012. Previous records: Belo Horizonte (Fernandes et al. 1988), and Serra de São José, MG (Maia \& Fernandes 2004). 

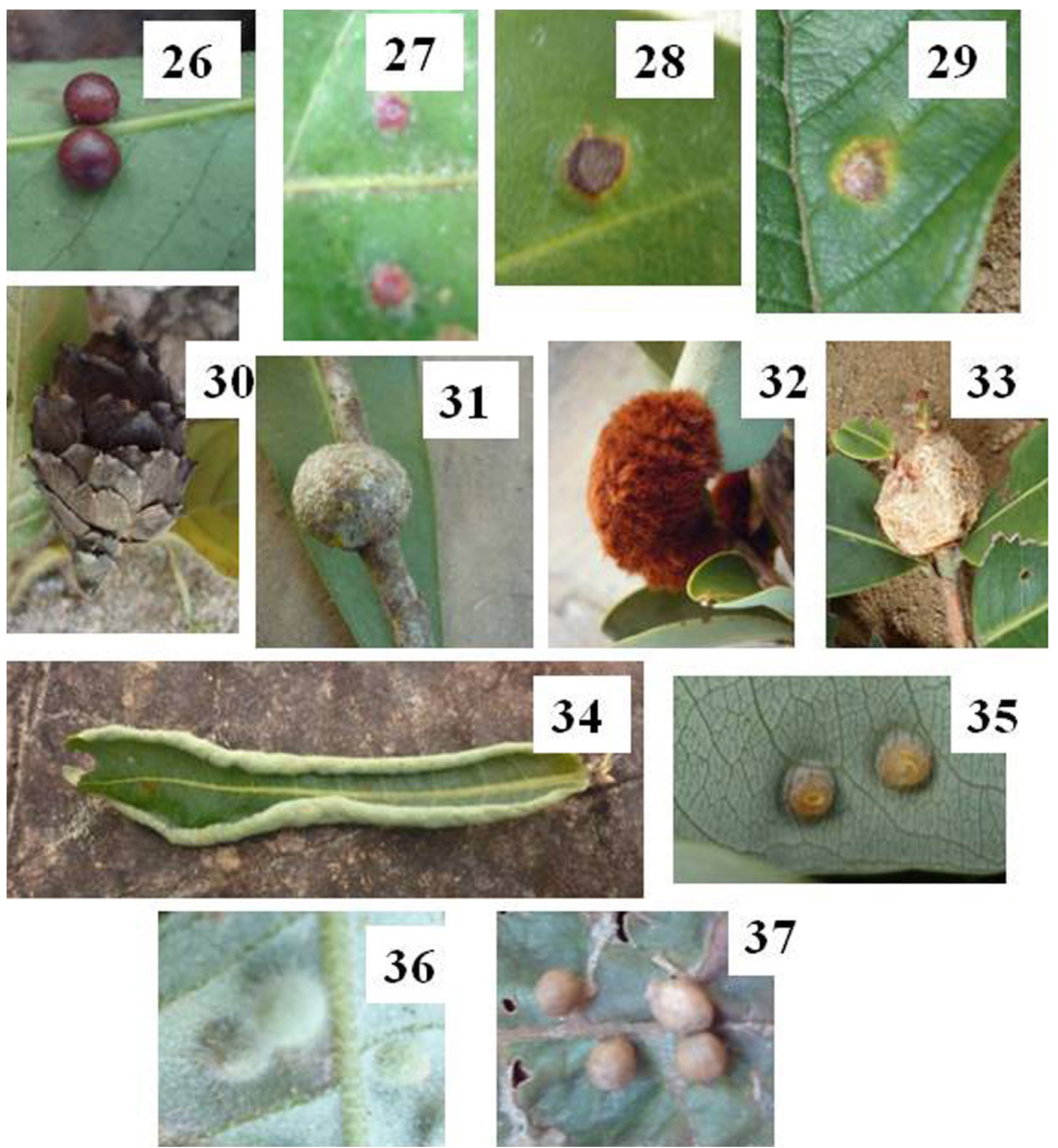

Figures 26-37. Insect galls from São Tomé das Letras: 26. Leaf gall on Maytenus sp.; 27. Leaf gall on Hippocratea sp.; 28. Leaf gall on Kielmeyera sp.; 29-30. Galls on Davilla brasiliensis: 29. Leaf gall, 30. Bud gall; 31. Stem swelling on Diospyros sp.; 32-35. Galls on Erythroxylum cf. suberosum: 32. Leaf gall, 33. Apical bud gall, 34. Marginal leaf roll, 35. Leaf gall; 36-37. Leaf galls on Croton sp.

Leaf gall, discoid, yellowish, green or brownish, glabrous, one-chambered (Figure 45). Galler: not determined. Localities: Cachoeira da Lua, Vale das Borboletas, Harmonia. Date: March/2012, June/2012. Previous records: Vale do Jequitinhonha (Fernandes et al. 1997), and Serra de São José, MG (Maia \& Fernandes 2004).

Leaf gall, globoid, yellowish, hairy, one-chambered (Figure 46). Galler: Cecidomyiidae (Diptera). Localities: Vale das Borboletas, Cachoeira da Lua. Date: December/2011, June/2012.
Leaf fold, yellowish or greenish, glabrous, one-chambered (Figure 47). Galler: Cecidomyiidae (Diptera). Locality: Cachoeira da Lua. Date: March/2012. Previous records: Serra do Cipó, MG (Coelho et al. 2009).

Leaf gall, spot-like, black, glabrous, one-chambered (Figure 48). Galler: not determined. Locality: Cachoeira da Lua. Date: $\operatorname{March} / 2012$. 
Maia, V.C.
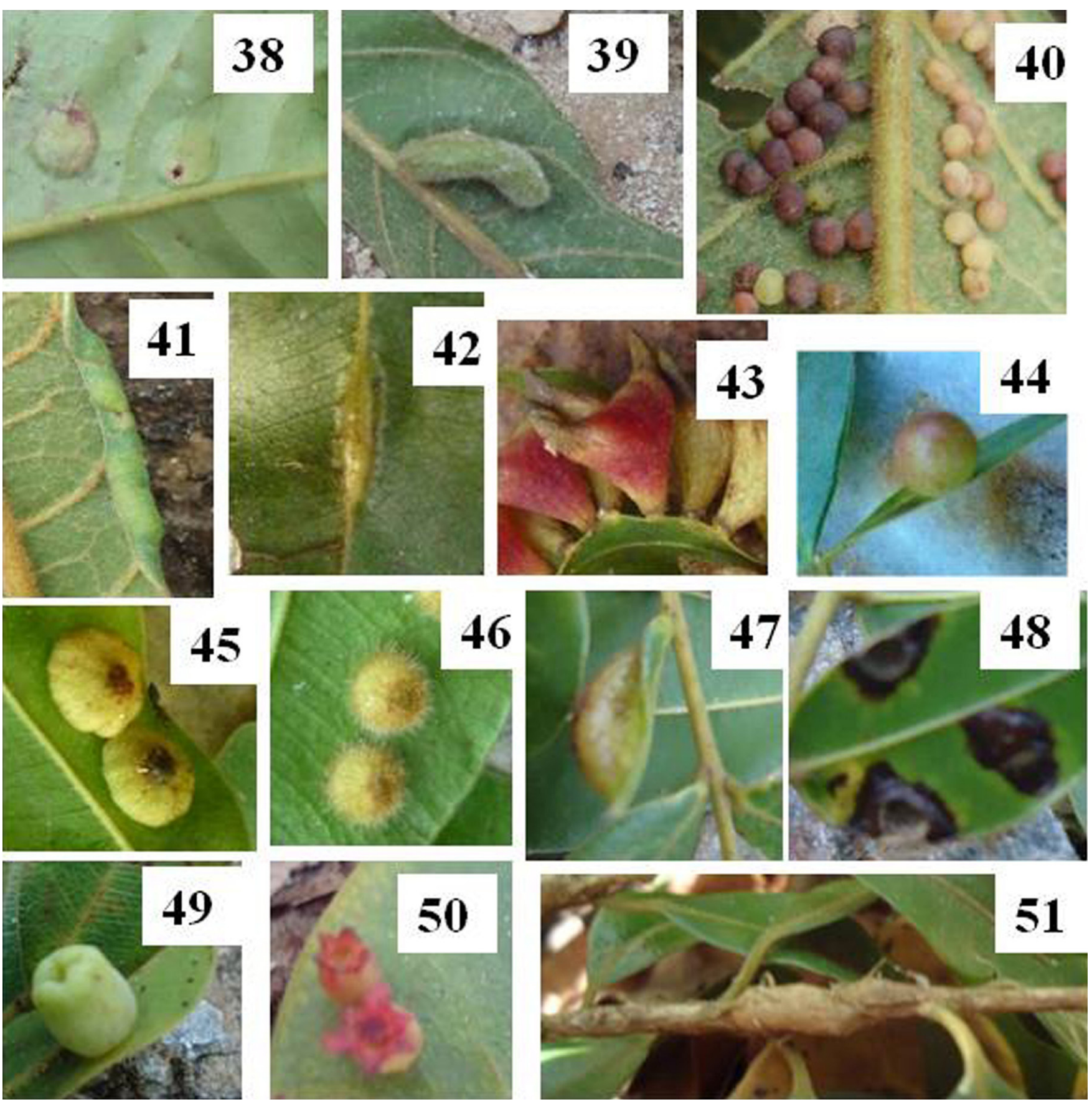

Figures 38-51. Insect galls from São Tomé das letras (MG): 38. Leaf gall on Pera sp.; 39-41. Galls on Andira sp.; 39. Vermiform gall; 40. Globoid gall; 41. Marginal roll; 42-51. Galls on Copaifera cf. langsdorfii; 42. Midvein swelling; 43. Horn-shaped gall; 44. Globoid gall (glabrous); 45. Discoid gall; 46. Globoid gall (hairy); 47. Leaf fold; 48. Spot-like gall; 49. Cylindrical gall; 50. Cylindrical gall; 51. Stem swelling.

Leaf gall, yellow or greenish, conical or cylindrical, glabrous, one-chambered (Figure 49). Galler: not determined. Localities: Vale das Borboletas, Cachoeira da Lua. Date: March/2012.

Leaf gall, cylindrical, reddish or yellowish, glabrous, one-chambered (Figure 50). Galler: not determined. Dweller: Thysanoptera. Locality: Gruta do Sobradinho. Date: September/2011.

Stem swelling, fusiform, brown, glabrous (Figure 51). Galler: not determined. Locality: Gruta do sobradinho. Date: September/2011.

Fernandes et al. (1988, 1997), Urso-Guimarães \& Scareli-Santos (2006), and Maia \& Souza (in press) described other galls on this host plant from Belo Horizonte (MG), Vale do Jequitinhonha (MG),
Santa Rita do Passa-Quatro (SP), and Ilha do Cabo Frio (Arraial do Cabo, RJ), respectively.

Dalbergia foliosa (Benth.) A. M. Carvalho $(\mathrm{n}=3)$

Stem swelling, fusiform, brown, glabrous, multi-chambered (Figure 52). Galler: Cecidomyiidae (Diptera). Localities: Ladeira do Amendoim, Cachoeira da Lua, Eubiose. Date: December/2011, March/2012, June/2012.

Leaf gall, globoid, yellowish or greenish, hairy, glabrous, onechambered (Figure 53). Galler: Cecidomyiidae (Diptera). Other dwellers: parasitoids (Hymenoptera). Locality: Cachoeira da Lua. Date: December/2011, March/2012. 

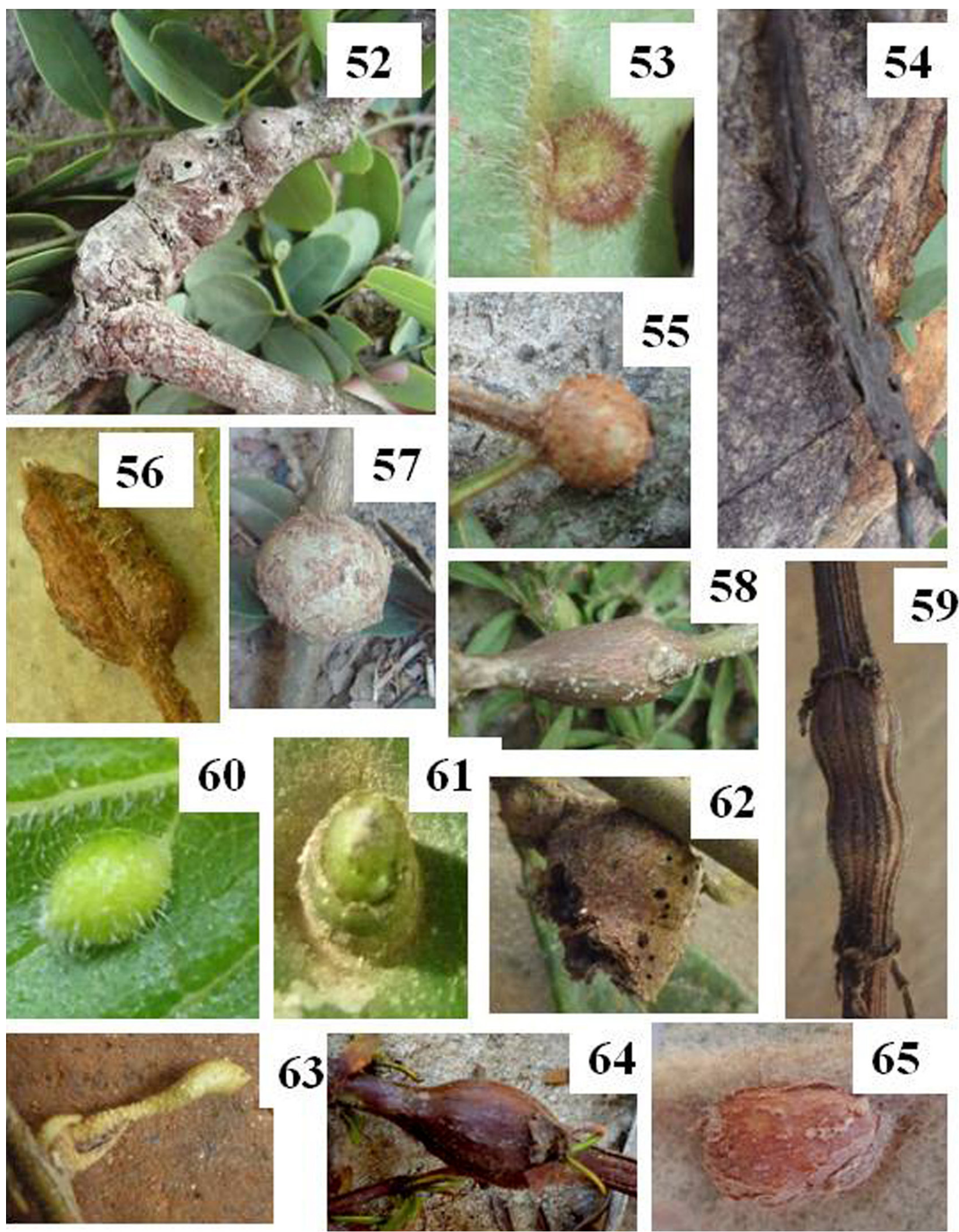

Figures 52-65. Insect galls of São Tomé das Letras (MG): 52-54. On Dalbergia foliosa; 52. Stem gall; 53. Leaf gall; 54. Stem gall (elongate); 55. Bud gall on Inga crassifolia; 56. Bud or stem gall on Inga edulis; 57. Stem swelling on Machaerium sp.; 58. Stem swelling on Stylosanthes gracilis; 59. Stem swelling on Hyptis recurvata; 60. Leaf or bud gall on Ocimum sp.; 61. Leaf gall on Ocotea cernua; 62. Bud gall on Ocotea sp.; 63. Aerial root swelling on Struthanthus sp.; 64. Stem swelling on Cuphea sp.; 65. Vein swelling on Byrsonima verbascifolia var. intermedia. 
Stem swelling, fusiform, elongate, dark brown, glabrous, multi-chambered (Figure 54). Galler: Lepidoptera. Other dwellers: parasitoids (Hymenoptera). Localities: Cachoeira da Lua, Gruta do Sobradinho. Date: December/2011.

Previous records: Maia (2001), Fernandes et al. (2001), Maia et al. (2008); Carneiro et al. (2009), Maia \& Oliveira (2010), and Maia et al. (in press) described some galls on Dalbergia spp. from Carapebus (RJ), Vale do Rio Doce (MG), Bertioga (SP), Cadeia do Espinhaço (MG), Ilha Grande (Angra dos Reis, RJ), and Santa Teresa (ES), respectively.

\section{Inga crassifolia Klotzsch ex Benth. $(\mathrm{n}=1)$}

Bud gall, globoid, brown, glabrous, one-chambered (Figure 55). Galler: not determined. Locality: Vale das Borboletas. Date: December/2011.

\section{Inga edulis Mart. ( $\mathrm{n}=1)$}

Bud or stem gall, fusiform, brown, glabrous, one-chambered (Figure 56). Galler: Cecidomyiidae (Diptera). Locality: Vale das Borboletas. Date: March/2012, June/2012. Previous records: UrsoGuimarães et al. (2003) recorded a leaf gall on this same host plant from Delfinópolis, MG.

Other galls on Inga spp. have been described by Fernandes et al. (1988) from Belo Horizonte (MG), Maia (2001) from Maricá (RJ), Julião et al. (2002) from Pantanal sul mato-grossense (MS); Maia \& Fernandes (2004) from Serra de São José (MG), Oliveira \& Maia (2005) from Grumari (Rio de Janeiro, RJ), Fernandes \& Negreiros (2006) from Aimorés (MG), Maia et al. (2008) from Bertioga (SP), Maia \& Oliveira (2010) from Ilha Grande (Angra dos Reis, RJ); Santos et al. (2010) from Goiânia (GO), Maia (2011) from Porto de Trombetas (Pará), Maia et al. (in press) from Santa Teresa (ES); and Maia (2012) from Itamonte (MG).

\section{Machaerium $\mathrm{sp} .(\mathrm{n}=1)$}

Stem swelling, globoid, brown, glabrous, one-chambered (Figure 57). Galler: Cecidomyiidae (Diptera). Locality: Cachoeira da Lua. Date: September/2011.

Previous records: Fernandes et al. (1988), Fernandes et al. (2001), Fernandes \& Negreiros (2006), Maia et al. (2008), Malves \& Frieiro-Costa (2012), and Maia et al. (in press) recorded stem galls on Machaerium spp. from Belo Horizonte (MG), Vale do Rio Doce (MG), Aimorés (MG), Bertioga (SP), Ingaí (MG), and Santa Teresa (ES).

\section{Stylosanthes gracilis Kunth. $(\mathrm{n}=1)$}

Stem swelling, fusiform, brownish or reddish, glabrous, onechambered (Figure 58). Galler: Cecidomyiidae (Diptera). Localities: Cachoeira da Lua, Gruta do Sobradinho. Date: March/2012.

Previous records: Itamonte, MG (Maia 2012). Other records: galls on Stylosanthes spp. were described by Coelho et al. (2009) from Serra do Cipó, MG.

Lamiaceae $(\mathrm{n}=2)$

\section{Hyptis recurvata Poit. $(\mathrm{n}=1)$}

Stem swelling, fusiform, brown, glabrous, one-chambered (Figure 59). Galler: Cecidomyiidae (Diptera). Localities: Gruta do Sobradinho, Eubiose. Date: June/2012.

Previous records: Galls on Hyptis spp. were described by Maia et al. (2008) from Bertioga (SP), Carneiro et al. (2009) from Cadeia do Espinhaço (MG), Coelho et al. (2009) from Serra do Cipó (MG), Santos et al. (2011) from Pernambuco, and Maia (2012) from Itamonte (MG).

\section{Ocimum sp. $(\mathrm{n}=1)$}

Leaf or bud gall, globoid, greenish, hairy (Figure 60). Galler: Cecidomyiidae (Diptera). Locality: ale das Borboletas. Date: December/2011.

Lauraceae $(\mathrm{n}=2)$

\section{Ocotea cernua (Nees) Mez. $(\mathrm{n}=1)$}

Leaf gall, conical, green, glabrous, one-chambered (Figure 61). Galler: not determined. Locality: Cachoeira da Lua. Date: June/2012.

Ocotea $\mathrm{sp} .(\mathrm{n}=1)$

Apical bud gall, ovoid, brown, glabrous, one-chambered (Figure 62). Galler: Cecidomyiidae (Diptera). Locality: Eubiose. Date: June/2012.

Previous records: Maia (2001), Fernandes et al. (2001), Julião et al. (2002), Maia et al. (2008), Carneiro et al. (2009), Bregonci et al. (2010), Saito \& Urso-Guimarães (2012), Maia et al. (in press); and Maia (2012) recorded other galls on Ocotea spp. from Carapebus and Maricá (RJ), Vale do Rio Doce (MG), Pantanal sul mato-grossense (MS), Bertioga (SP), Cadeia do Espinhaço (MG), Guarapari (ES), Luiz Antonio (SP), Santa Teresa (ES), and Itamonte $(\mathrm{MG})$, respectively.

Loranthaceae $(\mathrm{n}=1)$

Struthanthus sp. $(\mathrm{n}=1)$

Aerial root swelling, fusiform, brown, glabrous, one-chambered (Figure 63). Galler: Cecidomyiidae (Diptera). Locality: Eubiose. Date: June/2012.

Lythraceae $(\mathrm{n}=1)$

\section{Cuphea sp. $(\mathrm{n}=1)$}

Stem swelling, fusiform, brown, glabrous, one-chambered (Figure 64). Galler: Cecidomyiidae (Diptera). Localities: Cachoeira da Lua, Gruta do Sobradinho. Date: September/2011. Previous records: Itamonte, MG (Maia 2012). Other records: Carneiro et al. (2009) recorded a gall on Cuphea ericoides Cham. \& Schlech. from Cadeia do Espinhaço, MG.

\section{Malpighiaceae $(\mathrm{n}=5)$}

Byrsonima verbascifolia (L.) Rich. var. intermedia A. Juss. $(\mathrm{n}=1)$ Vein swelling, globoid, brown, woody, glabrous, one-chambered (Figure 65). Galler: not determined. Dwellers: Hymenoptera. Localities: Ladeira do Amendoim, Cachoeira da Lua, Gruta do Sobradinho. September/2011.

Byrsonima verbascifolia (L.) Rich. var. villosa Griseb ( $\mathrm{n}=1)$

Stem gall, globoid, brown, glabrous, one-chambered (Figure 66). Galler: not determined. Localities: Ladeira do Amendoim, Cachoeira da Lua, Gruta do Sobradinho. Date: March/2012.

Byrsonima verbascifolia (L.) Rich. $(\mathrm{n}=1)$

Leaf gall, conical, brown, micropubescent, glabrous, onechambered (Figure 67). Galler: Cecidomiidae (Diptera). Other dwellers: parasitoids (Hymenoptera). Localities: Gruta do Sobradinho, Ladeira do Amendoim, Harmonia. Date: September/2011, December/2011, March/2012. Previous records: Serra de São José, MG (Maia \& Fernandes 2004).

Previous records: Fernandes et al. (1997), Fernandes et al. (2001), Maia (2001), Julião et al. (2002), Urso-Guimarães et al. (2003), Maia \& Fernandes (2004), Oliveira \& Maia (2005), Urso-Guimarães \& Scareli-Santos (2006), Carneiro et al. (2009), Maia \& Oliveira (2010), Bregonci et al. (2010), Santos et al. (2011), Saito \& Urso-Guimarães (2012), and Maia et al. (in press) recorded several galls on Byrsonima 

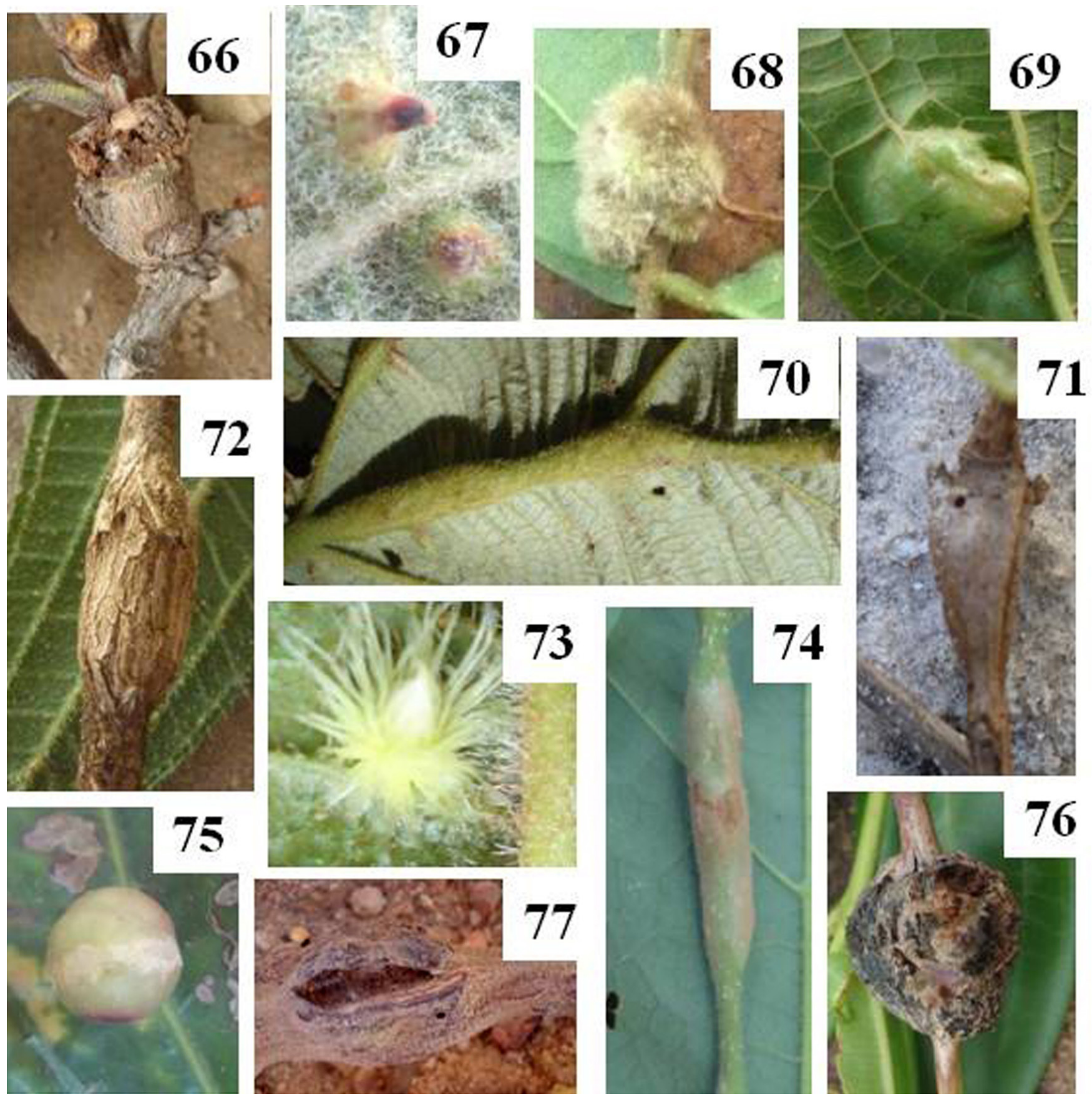

Figures 66-77. Insect galls of São Tomé das Letras (MG): 66. Stem gall on Byrsonima verbascifolia var. villosa; 67. Leaf gall on B. verbascifolia; 68. Bud gall on Mascagnia sp.; 69. Vein swelling on Mendocia sp.; 70. Midvein swelling on Luehea grandiflora; 71. Stem swelling on Melochia sp.; 72. Stem swelling on Aciotis cf.indecora; 73. Leaf gall on Clidemia sp.1; 74. Stem swelling on Clidemia sp.2; 75. Leaf gall on Miconia ligustroides; 76. Stem swelling on Miconia cf. theaezans; 77. Stem swelling on Miconia sp.1.

spp. from Vale do Jequitinhonha (MG), Vale do Rio Doce (MG), Carapebus and Maricá (RJ), Pantanal sul mato-grossense (MS), Delfinópolis (MG), Serra de São José (MG), Grumari (Rio de Janeiro, RJ), Santa Rita do Passa-Quatro (MG), Cadeia do Espinhaço (MG), Ilha Grande (Angra dos Reis, RJ), Guarapari (ES), Pernambuco, Luiz Antonio (SP), and Santa Teresa (ES), respectively.

Mascagnia sp. $(\mathrm{n}=1)$

Bud gall, ovoid, yellow, hairy, one-chambered (Figure 68) Galler: not determined. Localities: Vale das Borboletas, Eubiose. Date: September/2011, June/2012.

\section{Mendocia $\mathrm{sp} .(\mathrm{n}=1)$}

Vein swelling, globoid, greenish, glabrous, one-chambered (Figure 69). Galler: not determined. Locality: Eubiose. Date: June/2012.

Malvaceae $(n=3)$

Luehea grandiflora Mart. ( $\mathrm{n}=1)$

Midvein or petiole swelling, bulbous, greenish, glabrous, onechambered (Figure 70). Galler: Cecidomyiidae (Diptera). Locality: Cachoeira da Lua. Date: March/2012. 


\section{Melochia sp. $(\mathrm{n}=1)$}

Stem swelling, fusiform, brown, glabrous, one-chambered (Figure 71). Galler: not determined. Locality: Gruta do Sobradinho. Date: December/2011.

Waltheria indica L. $(\mathrm{n}=1)$

Leaf gall, globoid, yellow, hairy, glabrous, one-chambered. Galler: Cecidomyiidae (Diptera). Locality: Cachoeira da Lua. Date: June/2012.

Previous records: Santos et al. (2011) described other leaf gall on this plant from Pernambuco.

Melastomataceae $(\mathrm{n}=18)$

Aciotis cf. indecora (Bonpl) Triana ( $\mathrm{n}=2)$

Stem swelling, fusiform, brown, glabrous, one-chambered (Figure 72). Galler: not determined. Locality: Eubiose. Date: June/2012. Previous records: Itamonte, MG (Maia 2012).

Vein swelling, fusiform, green, glabrous, one-chambered. Galler: not determined. Locality: Eubiose. Date: June/2012. Previous records: Itamonte, MG (Maia 2012).

Clidemia sp. $1(\mathrm{n}=2)$

Leaf gall, globoid, red or yellow, hairy, glabrous, one-chambered (Figure 73). Galler: Cecidomyiidae (Diptera). Locality: Vale das Borboletas. Date: December/2011.

Bud gall, globoid, rugose, glabrous, one-chambered. Galler: not determined. Locality: Vale das Borboletas. Date: December/2011.

Clidemia sp. $2(\mathrm{n}=1)$

Stem swelling, fusiform, glabrous, one-chambered (Figure 74). Galler: not determined. Locality: Cachoeira da Lua. Date: March/2012.

Previous records: Fernandes et al. (2001), Maia et al. (2008), Maia et al. (in press), and Maia (2012) recorded galls on Clidemia spp. from Vale do Rio Doce (MG), Bertioga (SP), Santa Teresa (ES), and Itamonte (MG), respectively.

\section{Miconia ligustroides (DC.) Naudin ( $\mathrm{n}=1$ )}

Leaf gall, globoid, yellow, glabrous, one-chambered (Figure 75). Galler: not determined. Locality: Cachoeira da Lua. Date: $\operatorname{March} / 2012$.

\section{Miconia cf. theaezans Cogn. $(\mathrm{n}=1)$}

Stem swelling, fusiform, brown with black spot, glabrous, onechambered (Figure 76). Galler: not determined. Localities: Ladeira do Amendoim, Vale das Borboletas, Cachoeira da Lua, Gruta do Sobradinho, Harmonia. Date: December/2011, March/2012.

Miconia sp.1 (n=3)

Bud or stem swelling, globoid, brown, glabrous, one-chambered (Figure 77). Galler: not determined. Locality: Ladeira do Amendoim. Date: September/2011.

Bud or leaf gall, globoid, brownish, glabrous, one-chambered (Figure 78). Galler: not determined. Locality: Eubiose. Date: June/2012.

Leaf gall, globoid, reddish, micropubescent, glabrous, onechambered (Figure 79). Galler: Cecidomyiidae (Diptera). Locality: Eubiose. Date: June/2012.

Miconia sp.2 $(\mathrm{n}=1)$

Bud gall, globoid, green with red trichomes, one-chambered (Figure 80). Galler: Cecidomyiidae (Diptera). Locality: Cachoeira da Lua. Date: March/2012.
Miconia sp. $3(\mathrm{n}=2)$

Vein swelling, fusiform, green, glabrous, one-chambered (Figure 81). Galler: not determined. Locality: Vale das Borboletas. Date: June/2012.

Leaf gall, globoid, whitish, glabrous, one-chambered. Galler: not determined. Locality: Vale das Borboletas. Date: June/2012.

Previous records: Fernandes et al. (1997), Fernandes et al. (2001), Maia (2001), Maia \& Fernandes (2004), Urso-Guimarães \& Scareli-Santos (2006), Maia et al. (2008), Carneiro et al. (2009), Maia (2011), Malves \& Frieiro-Costa (2012), Saito \& Urso-Guimarães (2012), Maia et al. (in press), and Maia (2012) recorded several galls on Miconia spp. from Vale do Jequitinhonha (MG), Vale do Rio Doce (MG), Carapebus (RJ), Serra de São José (MG), Santa Rita do Passa-Quatro (SP), Bertioga (SP), Cadeia do Espinhaço (MG), Porto de Trombetas (Pará), Ingaí (MG), Luiz Antonio (SP), Santa Teresa (ES), and Itamonte (MG), respectively.

\section{Mouriri sp. $(\mathrm{n}=1)$}

Petiole swelling, bulbous, brown, glabrous, one-chambered (Figure 82). Galler: not determined. Locality: Gruta do Sobradinho. Date: March/2012.

\section{Tibouchina granulosa (Desr.) Cogn ( $\mathrm{n}=1)$}

Vein swelling, fusiform, green or brownish, glabrous, onechambered (Figure 83). Galler: Cecidomyiidae (Diptera). Locality: Cachoeira da Lua. Date: September/2011.

\section{Tibouchina cf. stenocarpa (DC.) Cogn. $(\mathrm{n}=1)$}

Leaf gall, burn-shaped, green, one-chambered (Figure 84). Galler: Lepidoptera. Locality: Cachoeira da Lua. Date: March/2012, June/2012. Previous records: Itamonte, MG (Maia 2012).

Previous records: Fernandes et al. (2001), Maia \& Fernandes (2004), Maia et al. (2008), Carneiro et al. (2009), and Maia et al. (in press) recorded several galls on Tibouchina spp. from Vale do Rio Doce (MG), Serra de São José (MG), Bertioga (SP), Cadeia do Espinhaço (MG), and Santa Teresa (ES), respectively.

Melastomataceae not determined $1(\mathrm{n}=1)$

Stem swelling, globoid, brown, glabrous, one-chambered (Figure 85). Galler: not determined. Locality: Ladeira do Amendoim. Date: September/2011.

Melastomataceae not determined $2(\mathrm{n}=1)$

Bud gall, globoid, green, glabrous, one-chambered (Figure 86). Galler: not determined. Locality: Ladeira do Amendoim. Date: June/2012.

Monimiaceae $(\mathrm{n}=1)$

Moriri sp. $(\mathrm{n}=1)$

Leaf gall, globoid, brown, hairy, one-chambered (Figure 87). Galler: not determined. Locality: Eubiose. Date: June/2012. Previous records: Itamonte, MG (Maia et al., in press).

Moraceae $(n=5)$

\section{Brosimum sp. $(\mathrm{n}=2)$}

Stem swelling, brown, ovoid, glabrous, multi-chambered (Figure 88). Galler: not determined. Dwellers: Hymenoptera. Locality: Ladeira do Amendoim. Date: December/2011.

Stem swelling, globoid, brown, glabrous, one-chambered (Figure 89). Galler: not determined. Locality: Gruta do Sobradinho. Date: December/2011. 

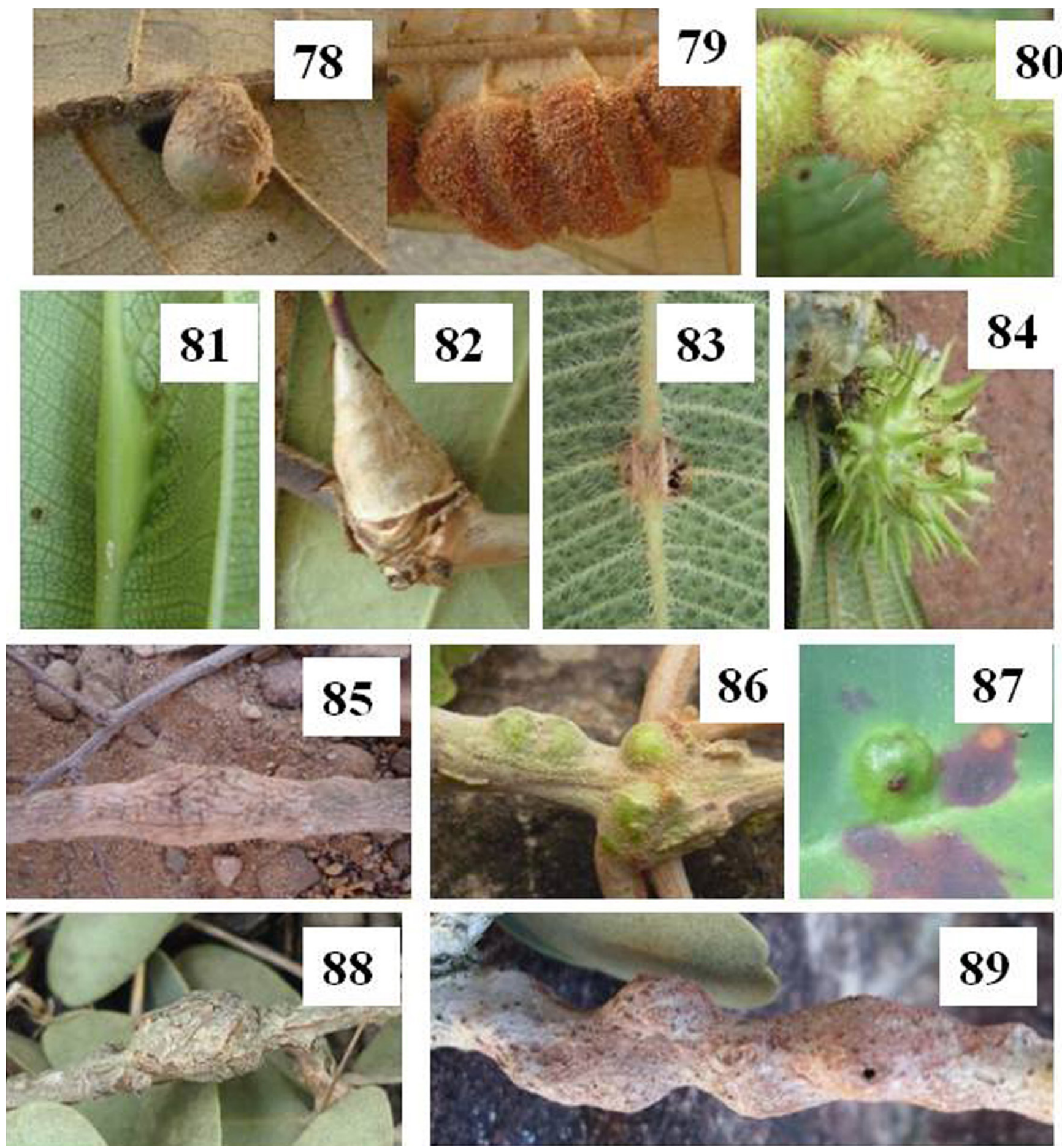

Figures 78-89. Insect galls of São Tomé das Letras (MG): 78. Bud or leaf gall on Miconia sp.1; 79. Leaf gall on Miconia sp.1; 80. Bud gall on Miconia sp.2; 81. Vein swelling on Miconia sp.3; 82. Petiole swelling on Mouriri sp.; 83. Vein swelling on Tibouchina granulosa; 84. Leaf gall on Tibouchina cf. stenocarpa; 85. Stem swelling on Melastomataceae not determined 1; 86. Bud gall on Melastomataceae not determined 2; 87. Leaf gall on Moriri sp.; 88. Stem swelling on Brosimum sp.; 89. Stem swelling on Brosimum sp.

Pseudolmedia macrophylla Trécul (n=3)

Leaf gall, globoid, brown, hairy, one-chambered (Figure 90). Galler: Cecidomyiidae (Diptera). Other dwellers: inquilines (Thysanoptera). Locality: Gruta do Sobradinho. Date: September/2011, March/2012.

Leaf gall, globoid, whitish, hairy, one-chambered (Figure 91). Galler: not determined. Locality: Gruta do Sobradinho. Date: September/2011.
Leaf gall, globoid, green-yellowish, glabrous, one-chambered (Figure 92). Galler: Hemiptera. Locality: Gruta do Sobradinho. Date: September/2011, March/2012.

Myrsinaceae $(\mathrm{n}=2)$

Rapanea cf. parvifolia (A. DC.) Mez (n=1)

Leaf gall, circular, green, glabrous, one-chambered (Figure 93). Galler: Cecidomyiidae (Diptera). Locality: Ladeira do Amendoim, 
Cachoeira da Lua, Gruta do Sobradinho. Date: December/2011, March/2012, June/2012.

\section{Rapanea $\mathrm{sp} .(\mathrm{n}=1)$}

Stem swelling, fusiform, brown, glabrous, one-chambered (Figure 94). Galler: Lepidoptera. Locality: Gruta do Sobradinho. Date: September/2011.

Previous records: Maia (2001), Maia \& Fernandes (2004), Maia et al. (2008), and Maia \& Oliveira (2010) recorded galls on Rapanea spp. from Maricá (RJ), Serra de São José (MG), Bertioga (SP), and Ilha Grande (Angra dos Reis, RJ).

Myrtaceae $(\mathrm{n}=17)$

Aulomyrcia tomentosa (Aubl.) DC. var. longipes $(\mathrm{n}=1)$

Bud gall, bulbous, green, micropubescent, one-chambered (Figure 95). Galler: Cecidomyiidae (Diptera). Locality: Cachoeira da Lua. Date: March/2012.

\section{Campomanesia pubescens (DC.) Berg. $(\mathrm{n}=1)$}

Stem gall, bulbous, reddish, glabrous, one-chambered. Galler: not determined. Locality: Vale das Borboletas. Date: December/2011, $\operatorname{March} / 2012$.

\section{Eugenia biflora L. $(\mathrm{n}=1)$}

Stem swelling, fusiform, brown, glabrous, one-chambered. Galler: Cecidomyiidae (Diptera). Locality: Ladeira do Amendoim. Date: June/2012.

\section{Eugenia punicifolia (Kunth.) DC. $(\mathrm{n}=1)$}

Stem swelling, fusiform, brown, glabrous, multi-chambered (Figure 96). Galler: Cecidomyiidae (Diptera). Locality: Ladeira do Amendoim. Date: March/2012.

\section{Eugenia sp. $(\mathrm{n}=1)$}

Bud gall apical, globoid, brown, glabrous, multi-chambered (Figure 97). Galler: not determined. Locality: Gruta do Sobradinho. Date: December/2011.

Previous records: Fernandes et al. (1988), Fernandes et al. (1997), Fernandes et al. (2001), Maia (2001), Julião et al. (2002), Maia \& Fernandes (2004), Oliveira \& Maia (2005), Urso-Guimarães $\&$ Scareli-Santos (2006), Maia et al. (2008), Carneiro et al. (2009), Coelho et al. (2009), Maia \& Oliveira (2010); Santos et al. (2011), Malves \& Frieiro-Costa (2012), Saito \& Urso-Guimarães (2012), Maia \& Souza (in press), Maia et al. (in press), and Maia (2012) recorded several galls on Eugenia spp. from Belo Horizonte (MG), Vale do Jequitinhonha (MG), Vale do Rio Doce (MG), Carapebus and Maricá (RJ), Pantanal sul mato-grossense (MS), Serra de São José (MG), Grumari (Rio de Janeiro, RJ), Santa Rita do Passa-Quatro (SP), Bertioga (SP), Cadeia do Espinhaço (MG), Serra do Cipó (MG), Ilha Grande (Angra dos Reis, RJ), Pernambuco, Ingaí (MG), Luiz Antonio (SP), Ilha do Cabo Frio (Arraial do Cabo, RJ), Santa Teresa (ES), and Itamonte (MG), respectively.

\section{Myrcia splendens (Sw.) DC. $(\mathrm{n}=2)$}

Stem swelling, fusiform, brown, glabrous, multi-chambered (Figure 98). Galler: Cecidomyiidae (Diptera). Locality: Cachoeira da Lua. Date: December/2011. Previous records: Serra do Cipó, MG (Coelho et al. 2009).

Bud gall, globoid, spongy, brown, glabrous, multi-chambered. Galler: not determined. Dwellers: Hymenoptera. Locality: Cachoeira da Lua. Date: December/2011.
Myrcia sylvatica (G. Mey) DC. $(\mathrm{n}=7)$

Leaf or bud gall, red, rugose, glabrous (Figure 99). Galler: Thysanoptera. Localities: Ladeira do Amendoim, Cachoeira da Lua, Gruta do Sobradinho. Date: December/2011, September/2011, March/2012, June/2012.

Marginal leaf roll, green, glabrous, one-chambered (Figure 100). Galler: Thysanoptera. Localities: Ladeira do Amendoim, Cachoeira da Lua, Gruta do Sobradinho, Harmonia, Eubiose. Date: September/2011, December/2011, March/2012, June/2012.

Leaf roll, green, glabrous, one-chambered (Figure 101). Galler: Cecidomyiidae (Diptera). Localities: Cachoeira da Lua, Vale das Borboletas, Eubiose. Date: December/2011, June/2012.

Stem swelling, fusiform, brown, multi-chambered (Figure 102). Galler: not determined. Dwellers: Hymenoptera. Locality: Cachoeira da Lua, Vale das Borboletas, Gruta do Sobradinho, Harmonia, Eubiose. Date: September/2011, March/2012. June/2012.

Leaf gall, globoid, green, glabrous, one-chambered (Figure 103). Galler: Cecidomyiidae (Diptera). Dwellers: Hymenoptera. Localities: Cachoeira da Lua, Harmonia, Eubiose. Date: December/2011, March/2012, June/2012.

Bud gall, conical, green-yellowish, glabrous, one-chambered (Figure 104). Galler: Cecidomyiidae (Diptera).Locality: Vale das Borboletas. Date: September/2011, June/2012.

Bud gall, horn-shaped, green, spongy, glabrous, multi-chambered (Figure 105). Galler: Cecidomyiidae (Diptera). Parasitoids (Hymenoptera), successors (Formicidae: adults, pupae and larvae). Localities: Cachoeira da Lua, Gruta do Sobradinho, Harmonia, Eubiose. Date: September/2011, December/2011, March/2012, June/2012.

\section{Myrcia sp. $(\mathrm{n}=1)$}

Bud gall, ovoid, green, glabrous, multi-chambered (Figure 106). Galler: not determined. Locality: Vale das Borboletas. Date: December/2011.

Previous records: Fernandes et al. (1988), Fernandes et al. (1997), Fernandes et al. (2001), Maia (2001), Maia \& Fernandes (2004), UrsoGuimarães \& Scareli-Santos (2006), Maia et al. (2008), Carneiro et al. (2009); Coelho et al. (2009), Santos et al. (2010), Malves \& FrieiroCosta (2012), Saito \& Urso-Guimarães (2012), Maia et al. (in press), and Maia (2012) recorded several galls on Myrcia spp. from Belo Horizonte (MG), Vale do Jequitinhonha (MG), Vale do Rio Doce (MG), Carapebus and Maricá (RJ), Serra de São José (MG), Santa Rita do Passa-Quatro (SP), Bertioga (SP), Cadeia do Espinhaço (MG), Serra do Cipó (MG), Goiânia (GO), Ingaí (MG), Luiz Antonio (SP), Santa Teresa (ES), and Itamonte (MG), respectively.

\section{Myrciaria floribunda (H. West ex Willd) O. Berg ( $\mathrm{n}=2$ )}

Stem swelling, fusiform, glabrous, one-chambered. Galler: Cecidomyiidae (Diptera). Localities: Vale das Borboletas, Eubiose. Date: June/2012.

Leaf gall, conical, green, glabrous, one-chambered (Figure 107). Galler: Cecidomyiidae (Diptera). Locality: Ladeira do Amendoim. Date: June/2012. Previous records: Santa Teresa, ES (Maia et al., in press).

Fernandes et al. (1997, 2001), Maia (2001), Maia \& Fernandes (2004), Maia \& Oliveira (2010), Bregonci et al. (2010), Maia et al. (in press), and Maia (2012) described some galls on Myrciaria spp. from Vale do Jequitinhonha (MG), Vale do Rio Doce (MG), Carapebus and Maricá (RJ), Serra de São José (MG), Ilha Grande (Angra dos Reis, RJ), Guarapari (ES), Santa Teresa (ES), and Itamonte (MG), respectively. 

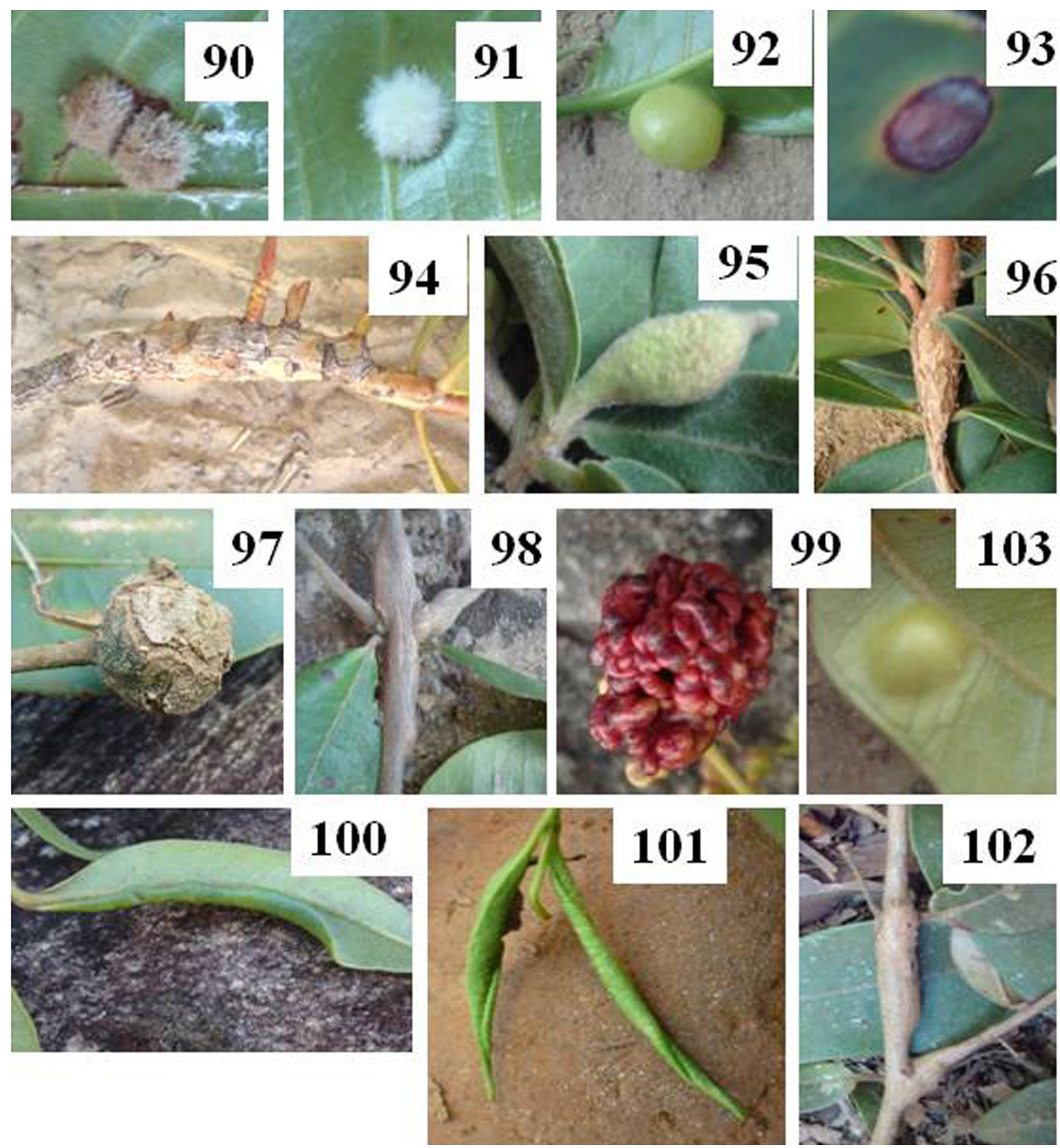

Figures 90-103. Insect galls of São Tomé das Letras: 90-92. Leaf galls on Pseudolmedia macrophylla, 90. Brown; 91. Whitish; 92. Green; 93. Leag gall on Rapanea cf. parvifolia; 94. Stem gall on Rapanea sp.; 95. Bud gall on Aulomyrcia tomentosa var. longipes; 96. Stem swelling on Eugenia punicifolia; 97. Bud gall on Eugenia sp.; 98. Stem swelling on Myrcia splendens; 99-103. Galls on Myrcia sylvatica: 99. Leaf or bud gall; 100. Leaf roll; 101. Marginal leaf roll; 102. Stem swelling; 103. Leaf gall.

Nyctaginaceae $(n=3)$

\section{Guapira sp. $(\mathrm{n}=1)$}

Leaf gall, globoid, green, glabrous, one-chambered (Figure 108). Galler: Cecidomyiidae (Diptera). Locality: Ladeira do Amendoim. Date: December/2011, March/2012.

Previous records: Galls on Guapira spp. have been described by Maia (2001) from Carapebus and Maricá (RJ), Oliveira \& Maia (2005) from Grumari (Rio de Janeiro, RJ), Maia et al. (2008) from
Bertioga (SP), Carneiro et al. (2009) from Cadeia do Espinhaço (MG), Maia \& Oliveira (2010) from Ilha Grande (Angra dos Reis, RJ), Bregonci et al. (2010) from Guarapari (ES), Maia \& Souza (in press) from Ilha do Cabo Frio (Arraial do Cabo, RJ), and Maia et al. (in press) from Santa Teresa (ES).

Neea $\mathrm{sp} .(\mathrm{n}=2)$

Rosette bud gall, green, glabrous (Figure 109). Galler: Cecidomyiidae (Diptera). Locality: Vale das Borboletas. Date: December/2011, June/2012. 

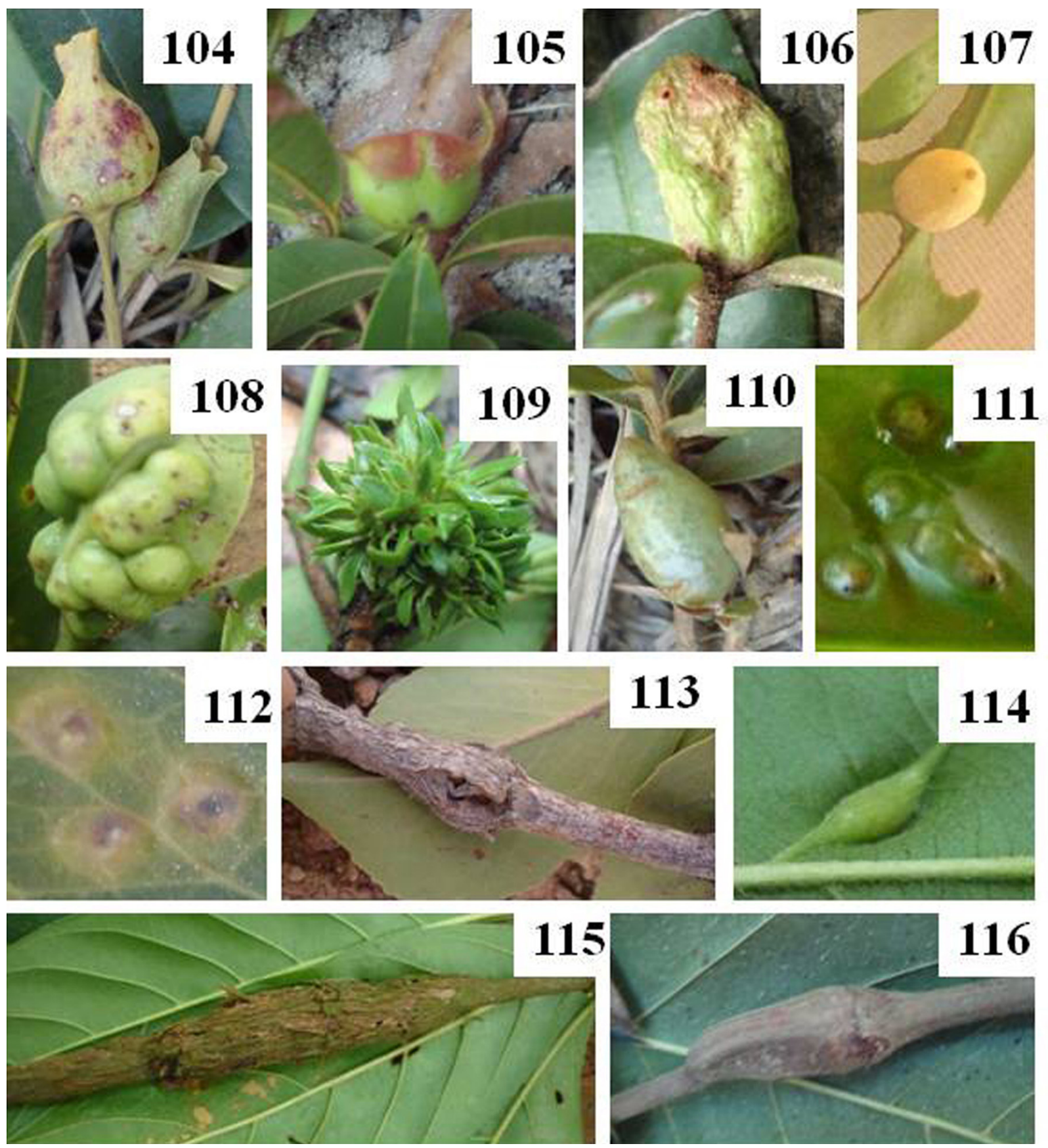

Figures 104-116. Insect galls of São Tomé das Letras (MG): 104-105. Galls on Myrcia sylvatica: 104. Bud gall (conical), 105. Bud gall (horn-shaped); 106. Bud gall on Myrcia sp.; 107. Leaf gall on Myrciaria floribunda; 108. Leaf gall on Guapira sp.; 109. Rosette bud gall on Neea sp.; 110. Bud gall on Turnera sp.; 111. Leaf gall on Piper sp.; 112-113. Galls on Roupala montana var. montana: 112. Leaf gall, 113. Stem swelling; 114-115. Galls on Amaioua intermedia var. brasiliana: 114. Vein swelling, 115. Stem swelling; 116. Stem swelling on Coussarea sp.

Leaf gall, globoid, red, hairy, one-chambered. Galler: Cecidomyiidae (Diptera). Locality: Vale das Borboletas. Date: December/2011.

Previous records: Julião et al. (2002), and Maia (2012) recorded galls on Neea spp. from Pantanal sul mato-grossense (MS), and Itamonte (MG).

Passifloraceae $(\mathrm{n}=1)$

\section{Turnera $\mathrm{sp} .(\mathrm{n}=1)$}

Bud gall, bursiform, green, glabrous, one-chambered (Figure 110). Galler: Hemiptera. Locality: Cachoeira da Lua. Date: September/2011.
Previous records: Maia (2011) described a leaf gall on Turnera sp. from Porto de Trombetas (Pará).

Piperaceae $(\mathrm{n}=1)$

\section{Piper sp. $(\mathrm{n}=1)$}

Leaf gall, circular, green, glabrous, one-chambered (Figure 111). Galler: Cecidomyiidae (Diptera). Locality: Vale das Borboletas. Date: September/2011, June/2012.

Previous records: Fernandes et al. (2001), Maia (2001), Maia \& Fernandes (2004), Oliveira \& Maia (2005), Maia et al. (2008), Santos et al. (2010), Maia et al. (in press), and Maia (2012) recorded 
galls on Piper spp. from Vale do Rio Doce (MG), Carapebus (RJ), Serra de São José (MG), Grumari (Rio de Janeiro, RJ), Bertioga (SP), Goiânia (GO), Santa Teresa (ES), and Itamonte (MG), respectively.

Proteaceae $(n=2)$

Roupala montana Aubl. var. montana $(\mathrm{n}=2)$

Leaf gall, circular, yellow, glabrous, one-chambered (Figure 112). Galler: Cecidomyiidae (Diptera). Other dwellers: parasitoids (Hymenoptera). Locality: Ladeira do Amendoim. Date:
September/2011, December/2011, March/2012, June/2012. Previous records: Luiz Antonio, SP (Saito \& Urso-Guimarães 2012).

Stem swelling, globoid, brown, glabrous, one-chambered (Figure 113). Galler: Cecidomyiidae (Diptera). Locality: Ladeira do Amendoim. Date: September/2011. Previous records: Luiz Antonio, SP (Saito \& Urso-Guimarães 2012).

Fernandes et al. (2001), and Maia (2012) described some galls on Roupala spp. from Vale do Rio Doce (MG), and Itamonte (MG), respectively.
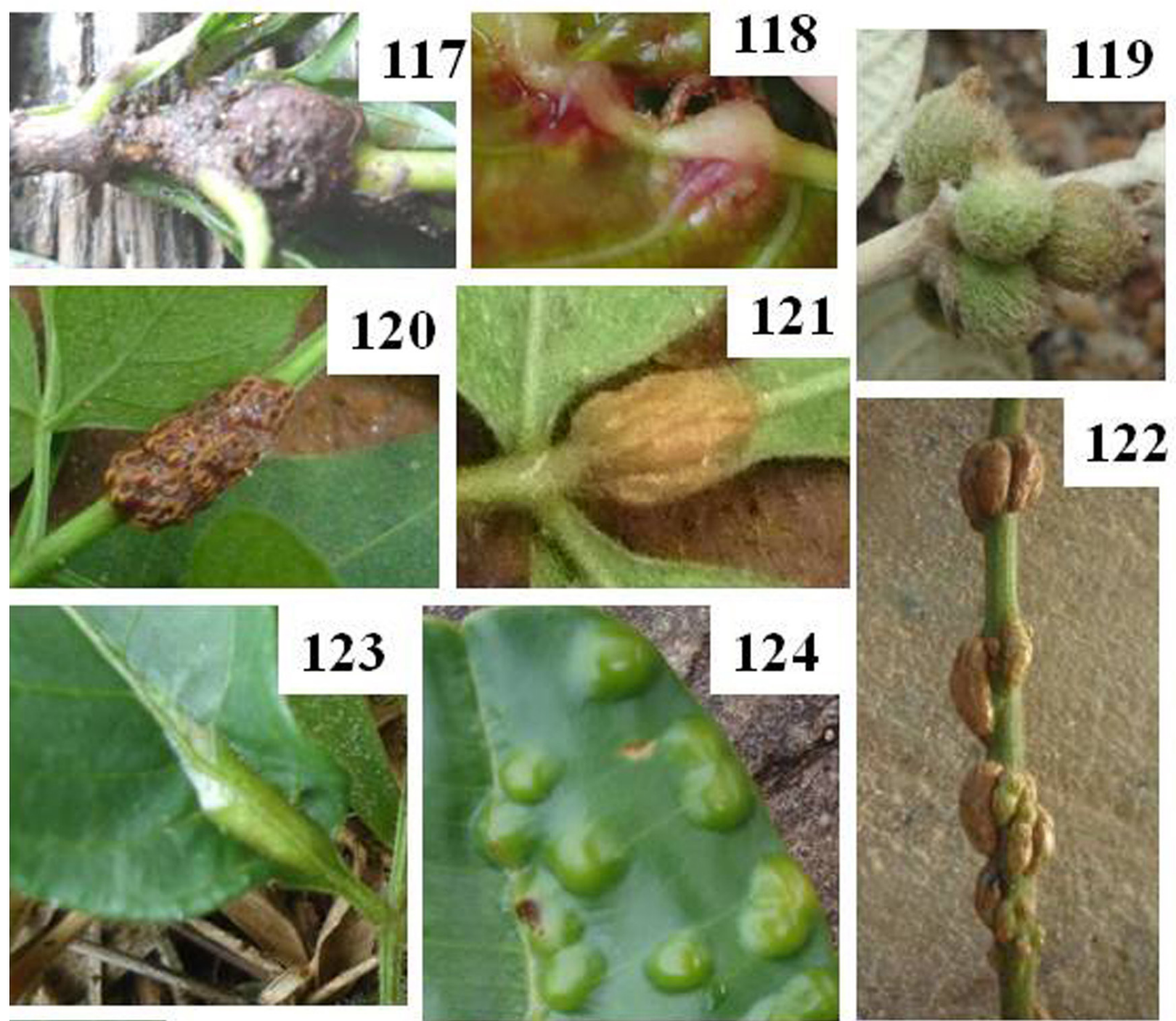

122
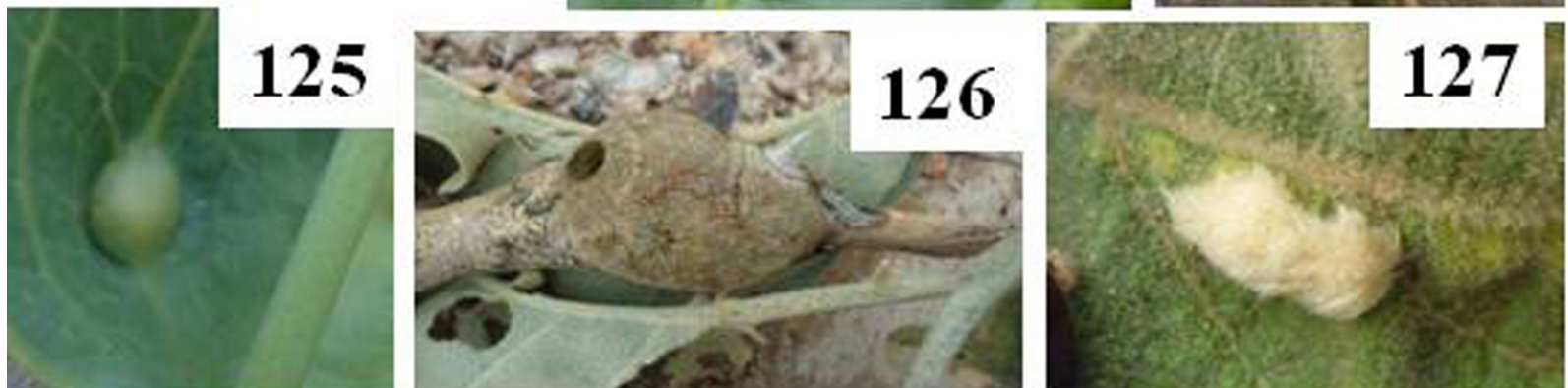

Figures 117-127. Insect galls of São Tomé das Letras (MG): 117. Stem swelling on Faramea sp.; 118. Leaf gall on Palicourea rigida; 119. Bud gall on Sabicea brasiliensis; 120-121. Galls on Serjania communis: 120. Stem swelling, 121. Petiole swelling; 122. Stem swelling on Serjania meridionalis; 123. Leaf gall on Serjania sp.; 124. Leaf gall, globoid on Manilkara sp.; 125. Vein swelling on Smilax cissoides; 126. Stem swelling on Solanum lycocarpum; 127. Leaf gall on Solanum sp. 
Rubiaceae $(\mathrm{n}=6)$

Amaioua intermedia var. brasiliana (A. Rich. ex DC.) $(\mathrm{n}=2)$

Vein swelling, fusiform, green, glabrous, one-chambered (Figure 114). Galler: not determined. Locality: Eubiose. Date: June/2012.

Stem swelling, fusiform, brown, glabrous, multi-chambered (Figure 115). Galler: not determined. Locality: Eubiose. Date: June/2012.

\section{Coussarea $\mathrm{sp} .(\mathrm{n}=1)$}

Stem swelling, fusiform, brown, glabrous, one-chambered (Figure 116). Galler: not determined. Locality: Vale das Borboletas. Date: September/2011.

\section{Faramea $\mathrm{sp} .(\mathrm{n}=1)$}

Stem swelling, globoid, brown, glabrous, one-chambered (Figure 117). Galler: not determined.Locality: Vale das Borboletas. Date: December/2011.

\section{Palicourea rigida Kunth. $(\mathrm{n}=1)$}

Leaf gall, globoid, red, glabrous, one-chambered (Figure 118). Galler: Cecidomyiidae (Diptera). Locality: Ladeira do Amendoim. Date: December/2011.

Previous records: Cadeia do Espinhaço (Carneiro et al. 2009). Other records: Fernandes et al. (1997) described other gall on this plant from Vale do Jequitinhonha (MG), Maia (2011), Maia et al. (in press), and Maia (2012) described galls on Palicourea spp. from Porto de Trombetas (Pará), Santa Teresa (ES), and Itamonte (MG), respectively.
Sabicea brasiliensis Wemham $(\mathrm{n}=1)$

Bud gall, green, globoid with a short apical projection, pubescent, one-chambered (Figure 119). Galler: not determined. Locality: Cachoeira da Lua. Date: March/2012.

Sapindaceae $(n=5)$

Serjania communis Cambess. $(\mathrm{n}=2)$

Stem swelling, fusiform, brown, glabrous, one-chambered (Figure 120). Galler: Cecidomyiidae (Diptera). Locality: Eubiose. Date: June/2012.

Petiole swelling, fusiform, brown, glabrous, one-chambered (Figure 121). Galler: Cecidomyiidae (Diptera). Locality: Eubiose. Date: June/2012.

\section{Serjania glutinosa Radlk. ( $\mathrm{n}=1)$}

Midvein swelling, fusiform, yellowish, one-chambered. Galler: Cecidomyiidae (Diptera). Locality: Ladeira do Amendoim. Date: March/2012.

\section{Serjania meridionalis Cambess $(\mathrm{n}=1)$}

Stem swelling, fusiform, brown, glabrous, one-chambered (Figure 122). Galler: Cecidomyiidae (Diptera). Locality: Eubiose. Date: June/2012.

\section{Serjania sp. $(\mathrm{n}=1)$}

Leaf gall, circular, green, glabrous, one-chambered (Figure 123). Galler: Cecidomyiidae (Diptera). Locality: Vale das Borboletas. Date: $M a r c h / 2012$.
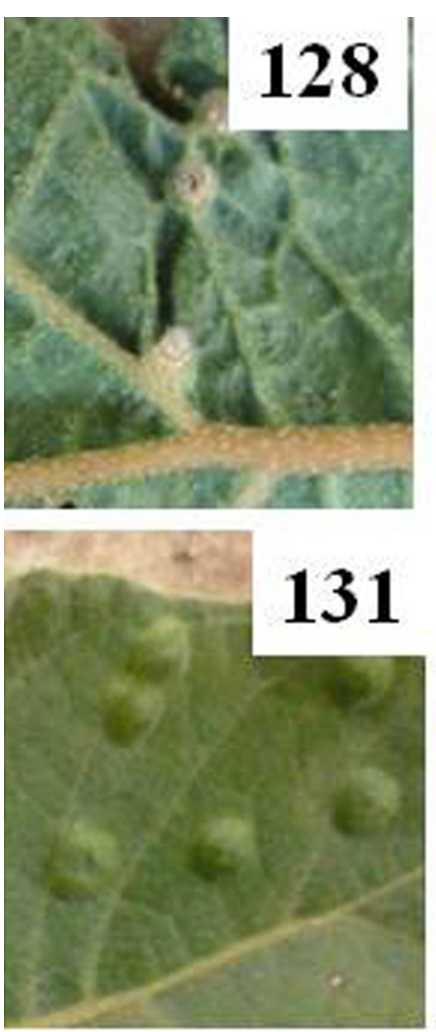
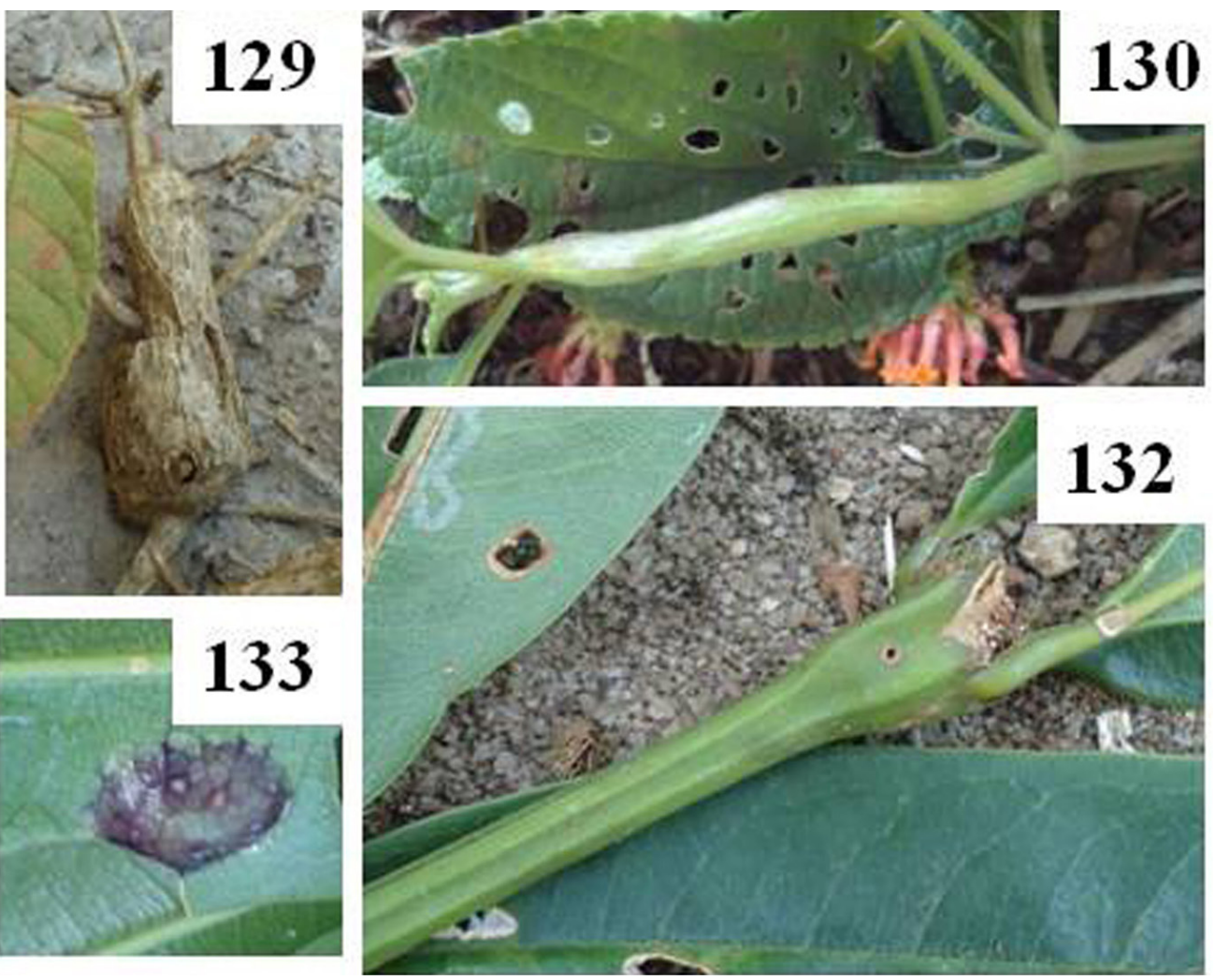

Figures 128-133. Insect galls of São Tomé das Letras (MG): 128. Leaf gall on Buettneria sp.; 129. Stem gall on Sterculiaceae not determined; 130. Stem swelling on Lantana camara; 131. Leaf gall on cf. Salvertia convallariodora; 132-133. Galls on Vochysia cf. maxima: 132. Stem swelling, 133. Leaf gall. 
Previous records: Fernandes et al. (1997), Fernandes et al. (2001), Julião et al. (2002), Coelho et al. (2009), Santos et al. (2010, 2011), Maia et al. (in press), and Maia (2012) recorded several galls on Serjania spp. from Vale do Jequitinhonha (MG), Vale do Rio Doce (MG), Pantanal sul mato-grossense (MS), Serra do Cipó (MG), Goiânia (GO), Pernambuco, Santa Teresa (ES), and Itamonte (MG), respectively.

Sapotaceae $(n=1)$

Manilkara $\mathrm{sp} .(\mathrm{n}=1)$

Leaf gall, globoid, green, glabrous, one-chambered (Figure 124). Galler: Cecidomyiidae (Diptera). Locality: Ladeira do Amendoim. Date: December/2011, March/2012.

Previous records: Galls on Manilkara spp. have been recorded by Oliveira \& Maia (2005) from Grumari (Rio de Janeiro, RJ), Maia \& Oliveira (2010) from Ilha Grande (Angra dos Reis, RJ), Bregonci et al. (2010) from Guarapari (ES), and Santos et al. (2011) from Pernambuco.

Siparunaceae $(n=1)$

\section{Siparuna guianensis Aubl. ( $\mathrm{n}=1)$}

Leaf gall, circular, green, glabrous, one-chambered. Galler: Cecidomyiidae (Diptera). Locality: Vale das Borboletas. Date: September/2011.

Previous records: Santos et al. (2010) described this gall from Goiânia (GO), Saito \& Urso-Guimarães (2012) recorded other galls on this host plant from Luiz Antonio (SP), Maia (2011) and Maia et al. (in press) described galls on Siparuna spp. from Porto de Trombetas (Pará), and Santa Teresa (ES), respectively.

Smilacaceae $(\mathrm{n}=1)$

\section{Smilax cissoides Mart. ex Griseb ( $\mathrm{n}=1)$}

Vein swelling, globoid, green, glabrous, one-chambered (Figure 125). Galler: Cecidomyiidae (Diptera). Locality: Gruta do Sobradinho. Date: December/2011.

Previous records: Maia (2001), Julião et al. (2002), UrsoGuimarães et al. (2003), Oliveira \& Maia (2005), Urso-Guimarães \& Scareli-Santos (2006), Maia et al. (2008), Carneiro et al. (2009), Coelho et al. (2009), Maia \& Oliveira (2010), Bregonci et al. (2010), Malves \& Frieiro-Costa (2012), and Maia et al. (in press) recorded leaf galls on Smilax spp. from Carapebus and Maricá (RJ), Pantanal sul mato-grossense (MS), Delfinópolis (MG), Grumari (Rio de Janeiro, RJ), Santa Rita do Passa-Quatro (SP), Bertioga (SP), Cadeia do Espinhaço (MG), Serra do Cipó (MG), Ilha Grande (Angra dos Reis, RJ), Guarapari (ES), Ingaí (MG), and Santa Teresa, respectively.

Solanaceae $(n=3)$

\section{Solanum lycocarpum A. St-Hil ( $\mathrm{n}=1)$}

Stem swelling, globoid, brown, glabrous, one-chambered (Figure 126). Galler: Collabismus clitellae Boheman (Curculionidae, Coleoptera). Locality: Gruta do Sobradinho. Date: December/2011. Previous records: Ingaí, MG (Malves \& Frieiro-Costa 2012).

Solanum $\mathrm{sp} .1(\mathrm{n}=1)$

Leaf gall, globoid, yellowish, hairy, one-chambered (Figure 127). Galler: not determined. Dwellers: Hymenoptera. Localities: Ladeira do Amendoim, Vale das Borboletas, Cachoeira da Lua. Date: December/2011, March/2012, June/2012.

Solanum sp. $2(\mathrm{n}=1)$
Midvein swelling, fusiform, green, glabrous, one-chambered. Galler: Cecidomyiidae (Diptera). Locality: Cachoeira da Lua. Date: March/2012.

Previous records: Several galls on Solanum spp. have been recorded by Fernandes et al. (2001) from Vale do Rio Doce, Maia (2001) from Carapebus and Maricá (RJ), Oliveira \& Maia (2005) from Grumari (Rio de Janeiro, RJ), Fernandes \& Negreiros (2006) from Aimorés (MG), Maia et al. (2008) from Bertioga (SP), Santos et al. (2011) from Pernambuco, Maia et al. (in press) from Santa Teresa (ES), and Maia (2012) from Itamonte (MG).

Sterculiaceae $(\mathrm{n}=2)$

\section{Buettneria sp. $(\mathrm{n}=1)$}

Leaf gall, globoid, yellowish or greenish, micropubescent, onechambered (Figure 128). Galler: not determined. Locality: Cachoeira da Lua. Date: December/2011.

Sterculiaceae not determined $(\mathrm{n}=1)$

Stem gall, fusiform, brown, woody, glabrous, one-chambered (Figure 129). Galler: Cecidomyiidae (Diptera). Dwellers: Hymenoptera. Locality: Gruta do Sobradinho. Date: September/2011.

Verbenaceae $(n=2)$

\section{Lantana camara $\mathrm{L} .(\mathrm{n}=1)$}

Stem swelling, fusiform, glabrous, one-chambered (Figure 130). Galler: Lepidoptera. Locality: Cachoeira da Lua. Date: March/2012.

Previous records: Fernandes et al. (2001), Maia (2001), Fernandes $\&$ Negreiros (2006), Santos et al. (2011), and Maia \& Souza (in press) recorded other galls on this host plant from Vale do Rio Doce (MG), Maricá (RJ), Aimorés (MG), Pernmabuco, and Ilha do Cabo Frio (Arraial do Cabo, RJ), respectively.

\section{Lantana $\mathrm{sp} .(\mathrm{n}=1)$}

Stem swelling, fusiform, brown, glabrous, one-chambered. Galler: Lepidoptera. Locality: Gruta do Sobradinho. Date: March/2012.

Previous records: Fernandes et al. (1997), Fernandes et al. (2001), Maia \& Fernandes (2004), Maia et al. (2008), Coelho et al. (2009), Carneiro et al. (2009), Maia \& Souza (in press), and Maia et al. (in press) recorded other galls on Lantana spp. from Vale do Jequitinhonha (MG), Vale do Rio Doce (MG), Bertioga (SP), Serra de São José (MG), Serra do Cipó (MG), Cadeia do Espinhaço (MG), Ilha do Cabo Frio (Arraial do Cabo, RJ), and Santa Teresa (ES), respectively.

Vochysiaceae $(n=4)$

\section{cf. Salvertia convallariodora A. St.-Hil $(\mathrm{n}=1)$}

Leaf gall, circular, green, glabrous, one-chambered (Figure 131). Galler: Cecidomyiidae (Diptera). Locality: Ladeira do Amendoim. Date: June/2012.

Vochysia cf. maxima Ducke $(\mathrm{n}=3)$

Marginal leaf roll, green, glabrous, one-chambered. Galler: Cecidomyiidae (Diptera). Locality: Harmonia. Date: March/2012.

Stem swelling, fusiform, green, glabrous, one-chambered (Figure 132). Galler: not determined. Locality: Harmonia. Date: March/2012.

Leaf gall, circular, black, glabrous, one-chambered (Figure 133). Galler: Cecidomyiidae (Diptera). Locality: Harmonia. Date: March/2012.

Previous records: Fernandes et al. (1997), and Maia (2012) described other galls on Vochysia spp. from Vale do Jequitinhonha (MG) and Itamonte (MG), respectively. 


\section{Discussion}

Several inventories of insect galls from Minas Gerais have been published (Fernandes et al. 1988, Fernandes et al. 1997, Fernandes et al. 2001, Urso-Guimarães et al. 2003, Maia \& Fernandes 2004, Fernandes \& Negreiros 2006, Carneiro et al. 2009, Coelho et al. 2009, Malves \& Frieiro-Costa 2012, Maia 2012). According to these inventories, the gall richness varies from 22 to 273 (Table 2), and the medium number of gall morphotypes per plant species from 1.15 to 1.96. São Tomé das Letras, with 152 gall morphotypes and a medium of 1.64 is the fourth richest investigated area of Minas Gerais.

Fabaceae, Melastomataceae, Myrtaceae, and Asteraceae were the plant families with the greatest richness of galls. All of them have been already indicated in other insect gall inventories as rich taxa in number of gall morphotypes (Table 3). So, the present study corroborates the previous knowledge about super host families (Veldtman \& McGeoch 2003), and the plant richness hypothesis (Fernandes 1992).

The super host genera were Copaifera L. (Fabaceae), Myrcia DC. ex. Guill. (Myrtaceae), and Miconia Ruiz \& Pav. (Melastomataceae). These three genera were already indicated in other gall inventories as super host (Campus Pampulha - Fernandes et al. (1988), Serra do Cipó - Coelho et al. (2009), Serra de São José - Maia \& Fernandes (2004), and Itamonte - Maia (2012)). Other cited genera were Bauhinia, Baccharis, Celtis, and Serjania (Serra do Cipó - Coelho et al. (2009), and Espinhaço - Carneiro et al. (2009)). Once more, this study corroborates the previous knowledge about super host genera.
The super host species were Copaifera langsdorfii Desf.(Fabaceae), Myrcia sylvatica (G. Mey) DC. (Myrtaceae), and Calophyllum brasiliense Cambéss (Calophyllaceae). Copaifera langsdorfii was already indicated in Campus Pampulha - Fernandes et al. (1988), and Serra de São José - Maia \& Fernandes (2004) as super host species. Bacccharis pseudomyriocephala (Espinhaço - Carneiro et al. (2009)), B. dracunculifolia (Serra do Cipó - Coelho et al. (2009)), Celtis brasiliensis (Serra do Cipó - Coelho et al. (2009)), and Protium heptaphyllum (Serra de São José - Maia \& Fernandes (2004)) are other indicated super hosts. Myrcia sylvatica, and Calophyllum brasiliense are indicated for the first time.

Leaves were the most galled plant organ, followed by stems, and buds. The same results were found in all other gall inventories of Minas Gerais, excepting by Espinhaço Range, where stems were the most galled plant organ (Carneiro et al. 2009). Stems were also indicated as the most galled plant organ in Vale do Rio Doce by Fernandes et al. (2001), but this result is not corrected. The authors considered vein and petiole as other plant organs, but technically they are part of the leaf, so if the values of vein and petiole are added, the total is $58 \%$, perpassing the stem. Leaves are the most galled plant organ throughout the world. It is a widespread pattern pointed by Felt (1940) and confirmed in this study.

The inducers are represented by Diptera, Lepidoptera, Hemiptera, Thysanoptera, Coleoptera, and Hymenoptera. These are the insect orders which comprise galling species. Cecidomyiidae (Diptera) were the most frequent and diversified gallers. This is true for all other gall inventories of Minas Gerais, where Cecidomyiidae have

Table 2. Distribution of the insect gall morphotypes, number of host plant species and medium number of gall morphotypes per plant species in some localities of Minas Gerais (Brazil).

\begin{tabular}{ccccc}
\hline Locality & $\begin{array}{c}\text { Nr. insect gall } \\
\text { morphotypes }\end{array}$ & $\begin{array}{c}\text { Nr. host plant } \\
\text { species }\end{array}$ & $\begin{array}{c}\text { Medium number of gall } \\
\text { morphotypes/ plant species }\end{array}$ & Reference \\
\hline Vale do Rio Doce & 273 & 139 & 1.96 & Fernandes et al. (2001) \\
Espinhaço Range & 241 & 142 & 1.69 & Carneiro et al. (2009) \\
Vale do Jequitinhonha & 236 & 134 & 1.76 & Fernandes et al. (1997) \\
São Tomé das Letras & 152 & 94 & 1.62 & Present study \\
Itamonte & 101 & 64 & 1.58 & Maia (2012) \\
Serra de São José & 137 & 73 & 1.87 & Maia \& Fernandes (2004) \\
Serra do Cipó & 91 & 43 & 1.78 & Coelho et al. (2009) \\
Boqueirão (Ingaí) & 57 & 22 & 1.32 & Malves \& Frieiro-Costa (2012) \\
Pampulha (Belo Horizonte) & 37 & 24 & 1.68 & Fernandes et al. (1988) \\
Fazenda Bulcão (Aimorés) & 29 & 19 & 1.21 & Fernandes \& Negreiros (2006) \\
Delfinópolis & 22 & & Urso-Guimarães et al. (2003) \\
\hline
\end{tabular}

Table 3. Distribution of the most galled host plant families per localities of Minas Gerais (Brazil).

\begin{tabular}{cccc}
\hline \multirow{2}{*}{ Localities of Minas Gerais } & \multicolumn{2}{c}{ Plant families } & References \\
\cline { 2 - 3 } & 1st & 2nd & Fernandes et al. (1988) \\
Pampulha (Belo Horizonte) & Fabaceae & Boraginaceae & Fernandes et al. (1997) \\
Vale do Jequitinhonha & Fabaceae & Malpighiaceae & Malves \& Frieiro-Costa (2012) \\
Boqueirão (Ingaí) & Asteraceae & Myrtaceae & Urso-Guimarães et al. (2003) \\
Delfinópolis & Fabaceae & Rubiaceae & Fernandes \& Negreiros (2006) \\
Fazenda Bulcão (Aimorés) & Fabaceae & Euphorbiaceae & Carneiro et al. (2009) \\
Espinhaço Range & Asteraceae & Melastomataceae & Coelho et al. (2009) \\
Serra do Cipó & Fabaceae & Myrtaceae & Fernandes et al. (2001) \\
Vale do Rio Doce & Asteraceae & Myrtaceae & Maia \& Fernandes (2004) \\
Serra de São José & Fabaceae & Myrtaceae & Maia (2012) \\
Itamonte & Asteraceae & Myrtaceae & Present study \\
São Tomé das Letras & Fabaceae & Melastomataceae &
\end{tabular}


been responsible for $93 \%$ to $73 \%$ of the insect galls. Cecidomyiidae is the most speciose galling taxa of the world with more than 6,000 species (Gagné 2010).

Globoid and fusiform galls predominated. Similar results were found in other Brazilian inventories, such as Espinhaço Range, MG (Carneiro et al. 2009), Pernambuco (Santos et al. 2001), Guarapari, ES (Bregonci et al. 2010), and Itamonte, MG (Maia 2012), suggesting that these are the most common gall shapes in Brazil.

The majority of the galls was glabrous $(86.18 \%)$ and onechambered $(90.8 \%)$ as in all other Brazilian galls inventories, excepting by Delfinópolis (MG), where hairy galls are more frequent than glabrous (Urso-Guimarães et al. 2003), but the difference is not significant.

The associated fauna included parasitoids (Hymenoptera), inquilines (Lepidoptera and Thysanoptera), successors (Formicidae, Hymenoptera), and predators (pseudoscorpions). The presence of parasitoids is very frequent in Brazilian surveys, being Hymenoptera the most important natural enemies of the gall midges (Maia 2001; Maia \& Azevedo 2009). The parasitoids frequence varies from $4 \%$ to $71 \%$ of the total of gall morphotypes (Table 4). The highest value was recorded to Pirenópolis, GO (Araujo et al. 2007), and the lowest to Porto de Trombetas (Maia 2012). São Tomé das Letras presented one of the lowest values. According to Fernandes \& Price (1992), parasitism is higher in mesic than in xeric habitats, because of longer maturation and hardening of the gall. This prediction is not confirmed in the present study.

Data on inquilines, successors, and predators are scarce in Brazilian gall inventories. In the present study, inquilines were rare, occurring in two gall morphotypes $(1.3 \%)$, being represented by Lepidoptera and Thysanoptera. In other Brazilian inventories, the frequency varies from $0 \%$ to $24.4 \%$ (Table 5 ), and other insects are cited as inquilines: Diptera, Lepidoptera, Coleoptera, Hemiptera, Psocoptera, and Thysanoptera. Comparing the results, the inquilines of São Tomé das Letras can be considered less frequent and little diversified.

Successors were recorded in two gall morphotypes in the present study (1.3\%), being represented by ants (Hymenoptera). The frequency of successors in Brazilian surveys is very low. The highest recorded value was 7.9\%, (Table 6). Successors can include Collembola, Psocoptera, and Thysanoptera also. The low frequency of successors is confirmed in this study. They are probably the less studied associated insects. In São Tomé das Letras, predators (pseudoscorpions) were recorded in a single gall morphotype $(0.6 \%)$. Similarly to the successors, they are also rare. In Brazilian

Table 4. Distribution of the frequency of parasitoids (Hymenoptera) in some Brazilian gall inventories.

\begin{tabular}{|c|c|c|}
\hline Localities & Frequency of parasitoids & Reference \\
\hline Pirenópolis (GO) & $71 \%$ & Araujo et al. (2007) \\
\hline Carapebus and Maricá & $60 \%$ & Maia (2001) \\
\hline Bertioga $(\mathrm{SP})$ & $48 \%$ & Maia et al. (2008) \\
\hline Serra de São José (MG) & $34 \%$ & Maia \& Fernandes (2004) \\
\hline Gurapari (ES) & $31 \%$ & Bregonci et al. (2010) \\
\hline Luiz Antônio (SP) & $23 \%$ & Saito \& Urso-Guimarães (2012) \\
\hline São Tomé das Letras (MG) & $12 \%$ & Present study \\
\hline Itamonte $(\mathrm{MG})$ & $6 \%$ & Maia (2012) \\
\hline Porto Trombetas (PA) & $4 \%$ & Maia (2011) \\
\hline
\end{tabular}

Table 5. Distribution of the frequency of inquilines in some Brazilian gall inventories.

\begin{tabular}{|c|c|c|c|}
\hline Localities & Frequency of inquilines & Inquilines identification & Reference \\
\hline Bertioga (SP) & $24.4 \%$ & $\begin{array}{c}\text { Diptera } \\
\text { Lepidoptera Coleoptera } \\
\text { Hemiptera Thysanoptera }\end{array}$ & Maia et al. (2008) \\
\hline Delfinópolis (MG) & $22.7 \%$ & $\begin{array}{c}\text { Diptera } \\
\text { Diplopoda } \\
\text { Psocoptera } \\
\text { Hemiptera }\end{array}$ & Urso-Guimarães et al. (2003) \\
\hline Carapebus and Maricá (RJ) & $17,6 \%$ & $\begin{array}{c}\text { Diptera } \\
\text { Hymenoptera } \\
\text { Lepidoptera Coleoptera }\end{array}$ & Maia (2001) \\
\hline Porto Trombetas (PA) & $17 \%$ & $\begin{array}{l}\text { Coleoptera } \\
\text { Diptera } \\
\text { Thysanoptera }\end{array}$ & Maia (2011) \\
\hline Guarapari (ES) & $10 \%$ & $\begin{array}{c}\text { Coleoptera Lepidoptera } \\
\text { Thysanoptera }\end{array}$ & Bregonci et al. (2010) \\
\hline Serra de São José (MG) & $4.7 \%$ & $\begin{array}{c}\text { Diptera } \\
\text { Coleoptera Lepidoptera } \\
\text { Hemiptera }\end{array}$ & Maia \& Fernandes (2004) \\
\hline Santa Rita do Passa Quatro (SP) & $2.8 \%$ & Diptera & Urso-Guimarães \& Scareli-Santos (2006) \\
\hline São Tomé das Letras (MG) & $1.3 \%$ & $\begin{array}{l}\text { Lepidoptera } \\
\text { Thysanoptera }\end{array}$ & Present study \\
\hline
\end{tabular}


Maia, V.C.

Table 6. Distribution of the frequency of successors in some Brazilian gall inventories.

\begin{tabular}{cccc}
\hline Localities & Frequency of successor & Successors identification & Reference \\
\hline Guarapari (ES) & $7.9 \%$ & Mites & Bregonci et al. (2010) \\
Bertioga (SP) & $4.7 \%$ & Psocoptera & Maia et al. (2008) \\
São Tomé das Letras (MG) & $1.3 \%$ & Collembola, Psocoptera, and Thysanoptera & Present study \\
Porto Trombetas (PA) & $1.3 \%$ & Hymenoptera & Maia (2011) \\
Carapebus and Maricá (RJ) & $1.0 \%$ & Psocoptera & Maia (2001) \\
Itamonte (MG) & $1.0 \%$ & Hymenoptera & Maia (2012) \\
\hline
\end{tabular}

Table 7. Distribution of the frequency of predators in some Brazilian gall inventories.

\begin{tabular}{cccc}
\hline Localities & $\begin{array}{c}\text { Frequency of } \\
\text { predators }\end{array}$ & Predators identification & Reference \\
\hline Carapebus and Maricá (RJ) & $4.0 \%$ & $\begin{array}{c}\text { Lestodiplosis spp. (Diptera, Cecidomyiidae) } \\
\text { Novohorus sp. (pseudoscorpions) }\end{array}$ & Maia (2001) \\
Guarapari (ES) & $2.6 \%$ & Hymenoptera (ants) & Bregonci et al. (2010) \\
Bertioga (SP) & $2.1 \%$ & Hymenoptera (ants) & Maia et al. (2008) \\
& $1.5 \%$ & Lestodiplosis sp. (Diptera: Cecidomyiidae) & Maia \& Fernandes (2004) \\
Serra de São José (MG) & $1.3 \%$ & Friebrigella sp. (Chloropidae: Diptera) & Maia (2011) \\
Porto Trombetas (PA) & $0.6 \%$ & Lestodiplosini (Diptera: Cecidomyiidae) & Present study \\
São Tomé das Letras (MG) & $0 \%$ & Pseudoscorpions & Maia (2012) \\
Itamonte (MG) & & &
\end{tabular}

inventories, the highest frequency is $4 \%$ (Table 7), and they are represented by ants (Formicidae, Hymenoptera), Lestodiplosis spp. (Diptera, Cecidomyiidae), Friebrigella sp. (Chloropidae: Diptera), and pseudoscorpions. This is the first record of pseudoscorpions in galls on Calophyllum brasiliense Cambéss (Calophyllaceae). They were previously recorded in galls on Myrtaceae.

The geographic distribution of six gall midges, Contarinia gemmae Maia, 2003; Dactylodiplosis heptaphylli Maia, 2004; Lopesia caulinaris Maia, 2003; Lopesia conspicua Maia, 2003; Lopesia elliptica Maia, 2003; and Lopesia linearis Maia, 2003 are widened to Minas Gerais.

Three other gall midges, Asphondylia serrata Maia, 2004, Lopesia similis Maia, 2004, and Myrciaryiamia admirabilis Maia, 2007, and one Tephritidae, Tomoplagia rudolphi (Lutz \& Lima, 1918) are recorded for the first time to São Tomé das Letras. These species were already known from other localities of Minas Gerais.

\section{Conclusions}

The present study corroborates the patterns of insect galls already known from Brazil, and for the first time indicates Myrcia sylvatica, and Calophyllum brasiliense as super host plants, and records the presence of pseudoscorpion in galls on Calophyllum brasiliense.

\section{Acknowledgments}

Thanks to CNPq for financial support (Proc.300237/2010-3) and to Dr. Bernardo Mascarenhas (Museu Nacional, UFRJ), Ms. Sueli Pereira (Museu Nacional, UFRJ) and Eduardo Barros (Museu Nacional, UFRJ) for field assistance.

\section{References}

ARAÚJO, W.S., GOMES-KLEIN, V.L. \& SANTOS, B.B. 2007. Galhas Entomógenas Associadas à Vegetação do Parque Estadual da Serra dos Pireneus, Pirenópolis, Goiás, Brasil.Revista Brasileira de Biociências 5 (supl. 1): 45-47.
BREGONCI, J.M., POLYCARPO, P.V. \& MAIA, V.C. 2010. Insect galls of the Parque Estadual Paulo César Vinha (Guarapari, ES, Brazil). Biota Neotrop. 10(1):265-274. http://dx.doi.org/10.1590/S167606032010000100023

CARNEIRO, M.A.A., BORGES, R.A.X., ARAÚJO, A.P.A. \& FERNANDES, G.W. 2009. Insetos indutores de galls da porção sul da Cadeia do Espinhaço, Minas Gerais, Brasil. Rev. Bras. Entomol. 53(4):570-592. http://dx.doi.org/10.1590/S0085-56262009000400007

COELHO, M.S., ALMADA, E.D., FERNANDES, G.W., CARNEIRO, M.A.A., SANTOS, R.M., QUINTINO, A.V. \& SANCHEZ-AZOFEIFA, A. 2009. Gall inducing arthropods from a seasonally dry tropical forest in Serra do Cipó, Brazil. Rev. Bras. Entomol. 53(3):404-414. http://dx.doi. org/10.1590/S0085-56262009000300015

FELT, E.P. 1940. Plant Galls and Gall Makers. Comstock Publishing Co., Ithaca, 364p.

FERNANDES, G.W. 1992. Plant family size and age effects on insular gallforming species richness. Global Ecol. Biogeogr. 2:71-74.

FERNANDES, G.W. \& NEGREIROS, D. 2006. A comunidade de insetos galhadores da RPPN Fazenda Bulcão, Aimorés, Minas Gerais, Brasil. Lundiana 7(2):111-120.

FERNANDES, G.W \& PRICE, P. 1992. The adaptive significance of the insect gall distribution: survivorship of species in xeric and mesic habitats. Oecologia 90:14-20. http://dx.doi.org/10.1007/BF00317803

FERNANDES, G.W., TAMEIRÃO-NETO, E. \& MARTINS, R.P. 1988. Ocorrência e caracterização de galhas entomógenas na vegetação do campus Pampulha da Universidade Federal de Minas Gerais. Rev. Bras. Zool. 5(1):11-29. http://dx.doi.org/10.1590/S0101-81751988000100002

FERNANDES, G.W., ARAÚJO, R.C., ARAÚJO, S.C., LOMBARDI, J.A., PAULA, A.S., LOYOLA JÚNIOR, R. \& CORNELISSEN, T.G. 1997. Insect galls from savanna and rocky fields of the Jequitinhonha valley, Minas Gerais, Brazil. Naturalia 22:221-244.

FERNANDES, G.W., JULIÃO, G.R., ARAÚJO, R.C., ARAÚJO, S.C., LOMBARDI, J.A., NEGREIROS, J.A., NEGREIROS, D. \& CARNEIRO, M.A.A. 2001. Distribution and morphology of insect galls of the Rio Doce Valley, Brazil. Naturalia 26:211-244. 
GAGNÉ, R.J.1989. The Plant-Feeding Gall Midges of North America. Ithaca, Cornell Univ. Press, 356 p.

GAGNÉ, R.J.,1994. The Gall Midges of the Neotropical Region. Ithaca: University Press. xiv, 352 p., 4 pls.

GAGNÉ, R.J. 2010. Update for a Catalog of the Cecidomyiidae (Diptera) of the world. http://www.ars.usda.gov/SP2UserFiles/Place/12754100/ Gagne_2010_World_Catalog_Cecidomyiidae.pdf. (last acess on I/2013).

JULIÃO, G.R., AMARAL, M.E.C. \& FERNANDES, G.W. 2002. Galhas de insetos e suas plantas hospedeiras no pantanal sul mato-grossense. Naturalia 27:47-74.

MAIA, V.C. 2001. The gall midges (Diptera, Cecidomyiidae) from three restingas of Rio de Janeiro State, Brazil. Rev. Bras. Zool. 18(2):583-629. http://dx.doi.org/10.1590/S0101-81752001000200028

MAIA, V. C. 2011. Characterization of insect galls, gall makers, and associated fauna of Platô Bacaba (Porto de Trombetas, Pará, Brazil). Biota Neotrop. 11(4):37-53. http://dx.doi.org/10.1590/S1676-06032011000400003

MAIA, V. C. 2012. In press. Insect galls of Itamonte (Minas Gerais, Brazil): characterization and occurrence. Biota Netrop.

MAIA, V.C. \&AZEVEDO, M.A.P. 2009. Micro-himenópteros associados com galhas de Cecidomyiidae (Diptera) em Restingas do Estado do Rio de Janeiro (Brasil). Biota Neotrop. 9(2):151-164. http://dx.doi.org/10.1590/ S1676-06032009000200015

MAIA, V.C. \& FERNANDES, G.W. 2004. Insect galls from Serra de São José (Tiradentes, MG, Brazil). Braz. J. Biol. 64(3):1-22.

MAIA, V.C. \& OLIVEIRA, J.C. 2010. Galhas de insetos da Reserva Biológica Estadual da Praia do Sul (Ilha Grande, Angra dos Reis, RJ). Biota Neotrop. 10(4):227-237. http://dx.doi.org/10.1590/S1676-06032010000400028

MAIA, V.C. \& SOUZA, M.C. In press. Insect galls of the xeric vegetation of Ilha do Cabo Frio (Arraial do Cabo, RJ, Brazil):characterization and occurrence. Biota neotrop.

MAIA, V.C., CARDOSO, L.J.T. \& BRAGA, J.M.A. In press. Insect galls from Atlantic Forest areas of Santa Teresa, Espírito Santo, Brazil. Bol. Mus. Biol. Mello Leitão.

MAIA, V.C., MAGENTA, M.A.C. \& MARTINS, S.E. 2008. Ocorrência e caracterização de galhas de insetos em áreas de restinga de Bertioga (São Paulo, Brasil). Biota Neotrop. 8(1):168-197. http://dx.doi.org/10.1590/ S1676-06032008000100020

MALVES, K. \& FRIEIRO-COSTA, F.A. 2012. List of plants with galls induced by insects from the UNILAVRAS/Boqueirão Biological Reserve, Ingaí, State of Minas Gerais, Brazil. Checklist 8(3):426-439.
MYERS, N., MITTERMEIER, R.A., MITTERMEIER, C.G., FONSECA, G.A.B. \& KET, J. 2000. Biodiversity hot-spots for conservation priorities. Nature 403:853-858. http://dx.doi.org/10.1038/35002501

OLIVEIRA, J.C. \& MAIA, V.C. 2005. Ocorrência e caracterização de galhas de insetos na restinga de Grumari (Rio de Janeiro, RJ, Brasil). Arq. Mus. Nac. 63(4):669-675.

SAITO, V.S. \& URSO-GUIMARÃES, M.V. 2012. Characterization of galls, insect galls and associated fauna of Ecological Station of Jataí (Luiz Antônio, SP). Biota Neotrop 12(3):01-09. http://dx.doi.org/10.1590/ S1676-06032012000300011

SANTOS, J.C., ALMEIDA-CORTEZ, J.S. \& FERNADES, G.W, 2001. Diversity of gall-inducing insects in the high altitude wetland forests in Pernambuco, Northeastern Brazil . Braz. J. Biol.. 71 (1): 47-56.

SANTOS, J.C., ALMEIDA-CORTEZ, J.C. \& FERNANDES, G.W. 2011. Richness of gall-inducing insects in the tropical dry forest (caatinga) of Pernambuco. Rev. Bras. Entomol. 55(1):45-54. http://dx.doi.org/10.1590/ S0085-56262011000100009

SANTOS, B.B., FERREIRA, H.D. \& ARAÚJO, W.S. 2010. Ocorrência e caraterização de galhas entomógenas em uma área de floresta estacional semi-decídua em Goiânia, Goiás, Brasil. Acta bot. bras. 24(1):243-249.

SHORTHOUSE, J.D. \& ROHFRITSCH, O., eds. 1992. Biology of Insect induced Galls. Oxford University Press, Oxford.

SHORTHOUSE, J.D.; WOOL, D. \& RAMAN, A. 2005. Gall-inducing insects - Nature's most sophisticated herbivores. Basic and Applied Ecology 6:407-411. http://dx.doi.org/10.1016/j.baae.2005.07.001

STONE, G. N. \& SCHÖNROGGE, K. 2003. The adaptive significance of insect gall morphology. Trends Ecology and Evolution 18: 512-522.

URSO-GUIMARÃES, M.V., SCARELI-SANTOS, C. \& BONIFÁCIOSILVA, A.C. 2003. Occurrence and characterization of entomogen galls from natural vegetation areas in Delfinópolis, MG, Brazil. Braz. J. Biol. 63(4):705-715. http://dx.doi.org/10.1590/S1519-69842003000400018

URSO-GUIMARÃES, M.V. \& SCARELI-SANTOS, C. 2006. Galls and gall makers in plants from the Pé-de-Gigante Cerrado Reserve, Santa Rita do Passa Quatro, SP, Brazil. Braz. J. Biol. 66(1B):357-369. http://dx.doi. org/10.1590/S1519-69842006000200018

VELDTMAN, R. \& McGEOCH, M.A. 2003. Gall-forming insect species richness along a non-scleromorphic vegetation rainfall gradient in South Africa: the importance of plant community composition. Austral Ecology 28:1-13. http://dx.doi.org/10.1046/j.1442-9993.2003.01234.x 